\title{
Comparative proteomic analysis reveals that exogenous 6-benzyladenine (6-BA) improves the defense system activity of waterlogged summer maize
}

\author{
Juan Hu, Baizhao Ren, Shuting Dong, Peng Liu, Bin Zhao and Jiwang Zhang* (0)
}

\begin{abstract}
Background: Exogenous 6-benzyladenine (6-BA) could improve leaf defense system activity. In order to better understand the regulation mechanism of exogenous 6-benzyladenine (6-BA) on waterlogged summer maize, three treatments including control (CK), waterlogging at the third leaf stage for 6 days (V3-6), and application of $100 \mathrm{mg}$ $\mathrm{dm}^{-3}$ 6-BA after waterlogging for 6 days (V3-6-B), were employed using summer maize hybrid DengHai 605 (DH605) as the experimental material. We used a labeling liquid chromatography-based quantitative proteomics approach with tandem mass tags to determine the changes in leaf protein abundance level at the tasseling stage.

Results: Waterlogging significantly hindered plant growth and decreased the activities of SOD, POD and CAT. In addition, the activity of LOX was significantly increased after waterlogging. As a result, the content of MDA and $\mathrm{H}_{2} \mathrm{O}_{2}$ was significantly increased which incurred serious damages on cell membrane and cellular metabolism of summer maize. And, the leaf emergence rate, plant height and grain yield were significantly decreased by waterlogging. However, application of 6-BA effectively mitigated these adverse effects induced by waterlogging. Compared with V3-6, SOD, POD and CAT activity of V3-6-B were increased by 6.9, 12.4, and 18.5\%, LOX were decreased by $13.6 \%$. As a consequence, the contents of $\mathrm{MDA}$ and $\mathrm{H}_{2} \mathrm{O}_{2}$ in $\mathrm{V} 3-6-\mathrm{B}$ were decreased by 22.1 and $17.2 \%$, respectively, compared to that of $\mathrm{V} 3-6$. In addition, the leaf emergence rate, plant height and grain yield were significantly increased by application of 6-BA. Based on proteomics profiling, the proteins involved in protein metabolism, ROS scavenging and fatty acid metabolism were significantly regulated by 6-BA, which suggested that application of 6-BA exaggerated the defensive response of summer maize at proteomic level.
\end{abstract}

Conclusions: These results demonstrated that 6-BA had contrastive effects on waterlogged summer maize. By regulating key proteins related to ROS scavenging and fatty acid metabolism, 6-BA effectively increased the defense system activity of waterlogged summer maize, then balanced the protein metabolism and improved the plant physiological traits and grain yield.

Keywords: Maize (Zea mays L.), Leaf, Proteomic, Tandem mass tags, Waterlogging; 6-benzyladenine

\footnotetext{
* Correspondence: jwzhang@sdau.edu.cn

State Key Laboratory of Crop Biology and College of Agronomy, Shandong

Agricultural University, Taian, Shandong 271018, People's Republic of China
}

(c) The Author(s). 2020 Open Access This article is distributed under the terms of the Creative Commons Attribution 4.0 International License (http://creativecommons.org/licenses/by/4.0/), which permits unrestricted use, distribution, and reproduction in any medium, provided you give appropriate credit to the original author(s) and the source, provide a link to the Creative Commons license, and indicate if changes were made. The Creative Commons Public Domain Dedication waiver (http://creativecommons.org/publicdomain/zero/1.0/) applies to the data made available in this article, unless otherwise stated. 


\section{Background}

Global warming has been unequivocally confirmed with many unprecedented observed changes such as increasing concentrations of greenhouse gases, warming of atmosphere, and extreme rainfall events over decades to millennia [1]. In China, the average surface temperature has increased by $1.1^{\circ} \mathrm{C}$, and the increase rate of temperature has reached $0.22^{\circ} \mathrm{C} / 10 \mathrm{a}$ over the last five decades [2]. The spatial and temporal distribution of rainfall has tended toward extremes and adverse climatic events, including waterlogging, drought, heat injury, low temperature, and freezing damage, are expected [3]. Such extreme events have done untold damages to environment, agricultural production, and long-term prospects of economy [4-7]. The frequency and intensity of extreme rainstorm events has increased in most parts of China since the 1980s, and the number of rainstorm days in the south of the Yangtze River and western, northern, and central parts of Henan Province has increased significantly since the 1990s [8]. Moreover, rainstorms and extreme precipitation events have tended to increase in south of $34^{\circ} \mathrm{N}$ [9]. Rainstorms are the most typical cause of waterlogging, which cause serious grain yield losses due to its paroxysmal and unpredictable nature [10]. Thus, the maize production system in HuangHuai-Hai Plain of China faces great challenges and risks against a backdrop of changing climate and increasing numbers of disastrous events. During the whole life cycle of maize production in Huang-Huai-Hai Plain, the frequency of waterlogging is as high as $30 \%$, especially during seedling to jointing and jointing to tasseling stages, which damages plant growth and increases grain yield loss significantly [9].

Maize is generally susceptible to waterlogging which may occur at different growth stages. Previous studies showed that waterlogging at different growth stages incurred diverse effects on the growth and yield of summer maize [11, 12]. To address waterlogging stress, plants would initiate a series of stress defensing processes going along with a wide-ranging changes of cell activities in plants. Plant defense system plays a key role in protecting plant from damaging under stresses [13]. As the antioxidant defense system was damaged under abiotic stresses, a significant increase in the accumulation of ROS in maize leaves was triggered which resulted in lipid peroxidation and membrane permeability [14]. At first, under adverse conditions (for examples, drought, salinity, cold, shade and so on), reactive oxygen species (ROS) are being generated as a secondary messenger to initiate subsequent defense reaction in plants which plays important roles in plant defense response to abiotic stress [15]. However, with the prolongation of stress duration, the ROS scavenging system was upset which disturbed the balance between production and quenching of ROS resulting in oxidative damages [16-20]. Antioxidant enzymes could effectively reduce the ROS damage on plants which activity may be directly related with plant tolerance to abiotic stresses [15].

6-Benzyladenine (6-BA) is a synthetic cytokinin (CTK)-like plant growth regulator that can significantly increase CTK levels in plants, of which levels are dramatically diminished under stress. CTKs are important growth-promoting compounds involved in inhibiting and scavenging active oxygen radicals, delaying leaf senescence. It has been reported that CTK improved the growth of plants exposed to stress by increasing superoxide dismutase activity and mitigating lipid peroxidation to maintain the balance between the production and scavenging of active oxygen radicals [21-23]. Applying 6-BA was conducive to minimize adverse effects of environmental stress such as drought, salt, low-temperature, and waterlogging stress [23-25]. In our previous studies, application of 6-BA effectively improved summer maize growth and increased the grain yield of summer maize under waterlogging stresses [26]. However, many studies have assessed the effects of 6-BA on plant growth, while only a few studies have investigated the regulation of molecular mechanism of exogenous 6-BA on the growth and development of waterlogged summer maize.

The proteome is the sum of all proteins expressed via gene transcription. Proteomics is a technique for studying the proteome to better represent life true characteristics at a deep level. It is significant to explore and discover the laws regulating biological activities, important physiological and pathological phenomena [27]. Proteomic analyses provide new insights to explore the potential of enhancing stress tolerance [28-30]. The changes of physiological metabolic processes at different plant stages can be studied by proteome analysis, because high-throughput proteomics studies cannot only reveal the mechanism of stress-related metabolic responses, but also reflect the specificity of different stress factors [30, 31]. Therefore, proteomics has attracted much academic attention. In particular, the different expression patterns of plant proteome provide insights into the regulation mechanisms and protective strategies of plants under different environmental stresses [32].

So far, quite a few studies have investigated the mechanism of waterlogging on summer maize, however, investigating the defense response mechanism of 6-BA on waterlogged summer maize at a level of proteins is still wanted. To mitigate this knowledge gap, a comparative proteomics study was applied to investigate the molecular mechanisms of 6-BA on waterlogged summer maize. Proteins related to plant defense system activity whose abundance was regulated by 6-BA were identified. Combining with the physiological traits, this study 
contributed to a better understanding of how exogenous 6-benzyladenine (6-BA) regulated the defense system activity in waterlogged summer maize.

\section{Results}

Plant growth and grain yield of summer maize

The field experiment results showed that waterlogging significantly impeded the plant growth by decreasing leaf emergence rate and plant height. At VT, the plant height of waterlogging treatment (V3-6) was decreased by $25.9 \%$, compared to that of CK. However, spraying 6-BA was conducive to mitigate the decrease of plant height of waterlogged maize. The plant height of V3-6-B was increased by $12.3 \%$, compared to that of V3-6. The leaf emergence rate was delayed by waterlogging, the V6, V9, V12 growth and development stages were delayed by 1,2 , 2 days in V3-6, respectively, compared with CK. In V3-6$\mathrm{B}$, the V6, V9, V12 growth and development stages were all delayed by 1 days, compared with that of CK (Fig. 1).

\section{ROS scavenging system}

The activities of SOD, POD, and CAT were significantly decreased after waterlogging. The reduction in the activities of SOD, POD and CAT were around 14.0, 16.3 and $41.0 \%$, respectively, compared to those of CK. The MDA content was increased by $38 \%$ in V3-6, compared to that of CK. However, application of 6-BA alleviated waterlogging stress on the activities of antioxidative enzymes. Applying 6-BA resulted in significant and substantial increases in SOD (around 6.9\%), POD (around 12.4\%), and CAT (around 18.5\%), compared to those of the waterlogging treatment. And also, application of 6-BA alleviated waterlogging stress on MDA content, with $22.1 \%$ decrease of MDA content in V3-6-B, compared to that of V3-6 (Fig. 2).

\section{LOX activity and $\mathrm{H} 2 \mathrm{O} 2$ content}

In this study, the LOX activity was increased by $23.4 \%$ after waterlogging, however, this increase was effectively inhibited by application of 6-BA. In addition, the $\mathrm{H}_{2} \mathrm{O}_{2}$

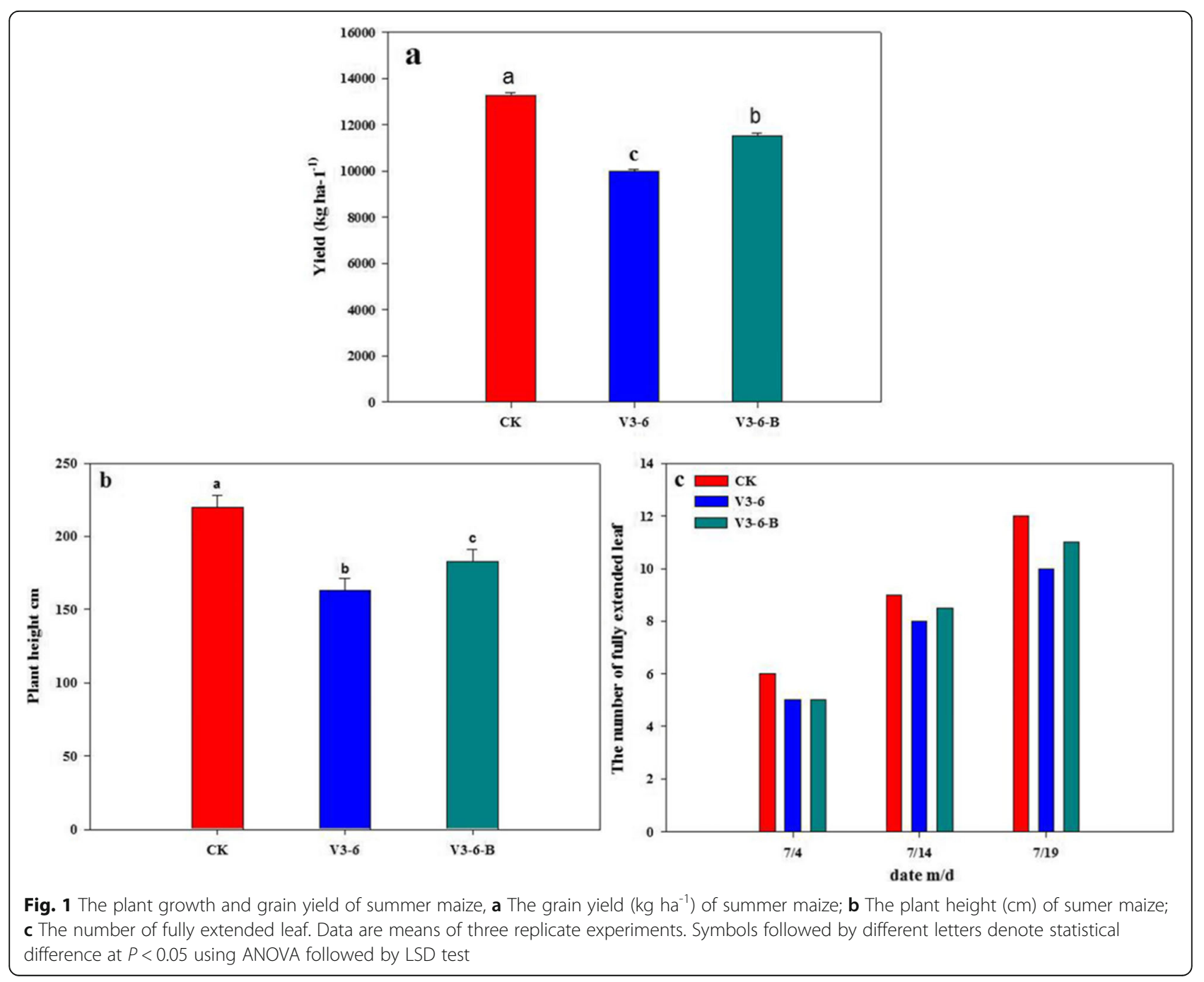



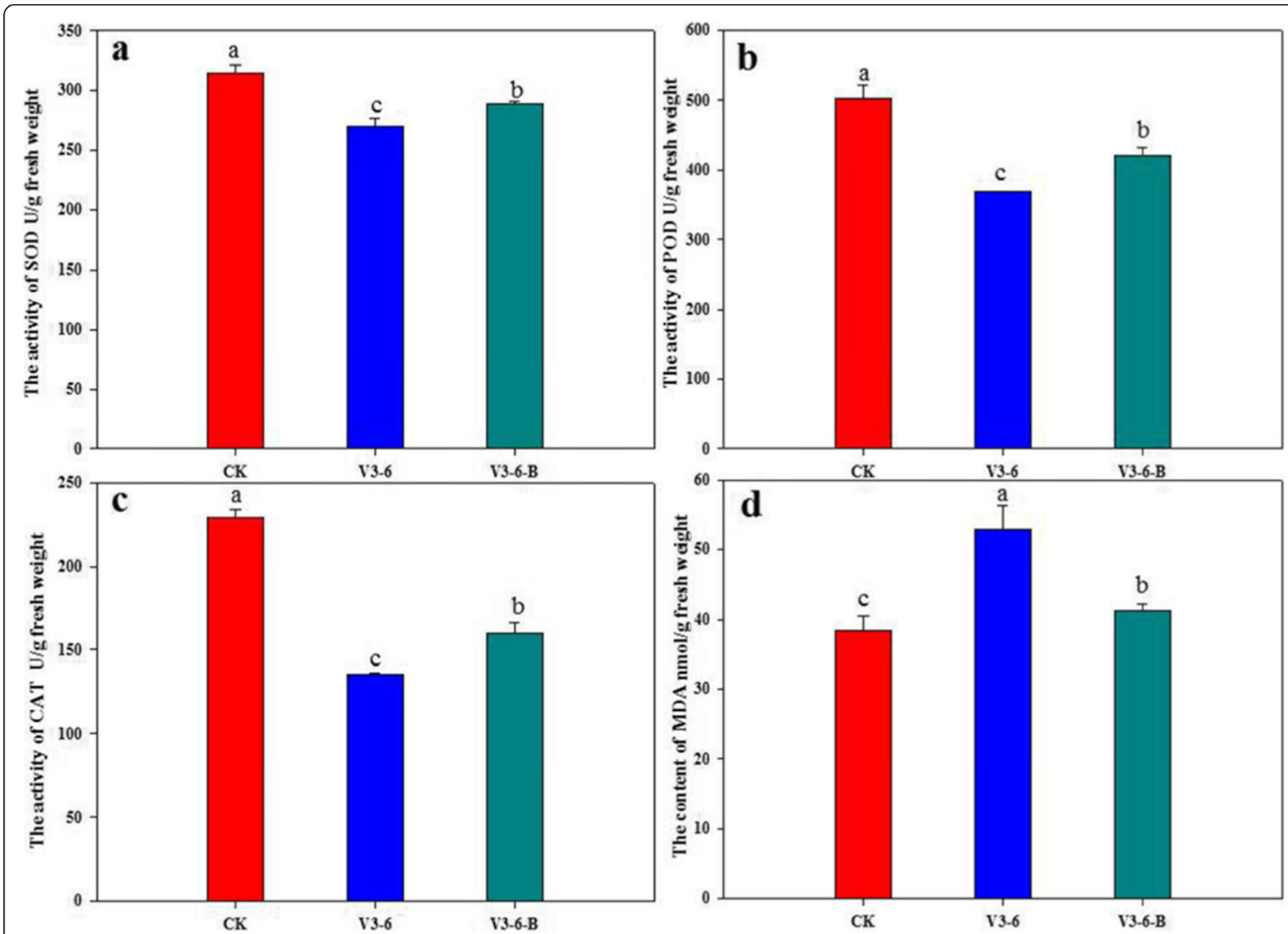

Fig. 2 The enzymes (SOD, POD, CAT) activity and MDA content in different treatments, a The activity of SOD (U/g fresh weight) in summer maize; $\mathbf{b}$ The activity of POD (U/g fresh weight) in summer maize; c The activity of CAT (U/g fresh weight) in summer maize; $\mathbf{d}$ The content of MDA (nmol/g fresh weight) in summer maize. Data are means of three replicate experiments. Symbols followed by different letters denote statistical difference at $P<0.05$ using ANOVA followed by LSD test

content in V3-6 treatment was about $52.3 \%$ higher than in CK, while that of V3-6-B was about $17.2 \%$ lower than that of V3-6 (Fig. 3).

\section{Identification of differentially accumulated proteins}

The result of quality detection by mass spectrometry showed that the peptide mass error central distributes in below 10 ppm scopes and most peptide distributed in $8-$ 20 amino acid residues (Additional file 1: Figure S1). In addition, the results of Pearson's correlation of quantitation among treatments also indicated that our samples reached the requirements for further study (Additional file 1: Figure S2). In total, 5322 leaf proteins were detected, and 4437 were quantified, using the criteria that proteins with a fold-change $\geq 1.5$ were considered as differentially abundant proteins. One hundred fifty two and 28 leaf proteins were identified as significantly up-regulated and down-regulated proteins under waterlogging stress (V36), 48 and 67 proteins were identified as significantly upregulated and down-regulated proteins in V3-6-B treatment, respectively, and 222 proteins were differentially abundant between V3-6 and V3-6-B (Additional file 1: Figure S3). Among the differentially accumulated proteins, about $4 \%$ were involved in antioxidant activity, $10 \%$ were involved in responding to stimulus, and 30\% were related to metabolic process, these proteins were mostly related with plant defense system (Additional files 2 and 3).

\section{Bioinformatic analysis of differentially abundant proteins} GO annotation was performed to identify the significantly enriched GO functional groups of differentially abundant proteins. Comparing with $\mathrm{CK}$, The up-accumulated proteins of V3-6 and V3-6-B treatments were strongly enriched in defense-related proteins including "plant response to stress", "response to toxic substance", "response to oxidative stress", "response to stimulus", "hydrogen peroxide metabolic process" and mainly had functions in peroxidase activity, tetrapyrrole binding and oxidoreductase activity. These proteins played key roles in protecting plant from damages (Fig. 4). 

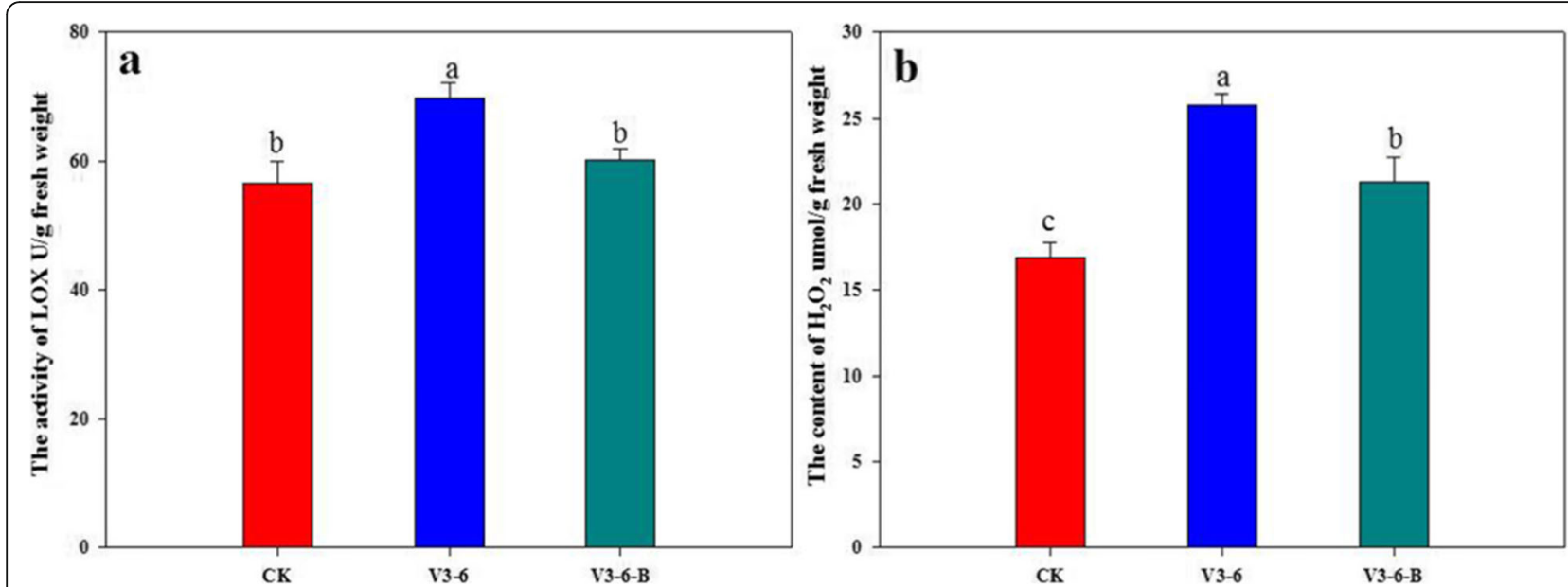

Fig. 3 The LOX activity and $\mathrm{H}_{2} \mathrm{O}_{2}$ content in different treatments, a The activity of LOX ( $\mathrm{U} / \mathrm{g}$ fresh weight); $\mathbf{b}$ The content of $\mathrm{H}_{2} \mathrm{O}_{2}$ (umol/g fresh weight). Data are means of three replicate experiments. Symbols followed by different letters denote statistical difference at $P<0.05$ using ANOVA followed by LSD test

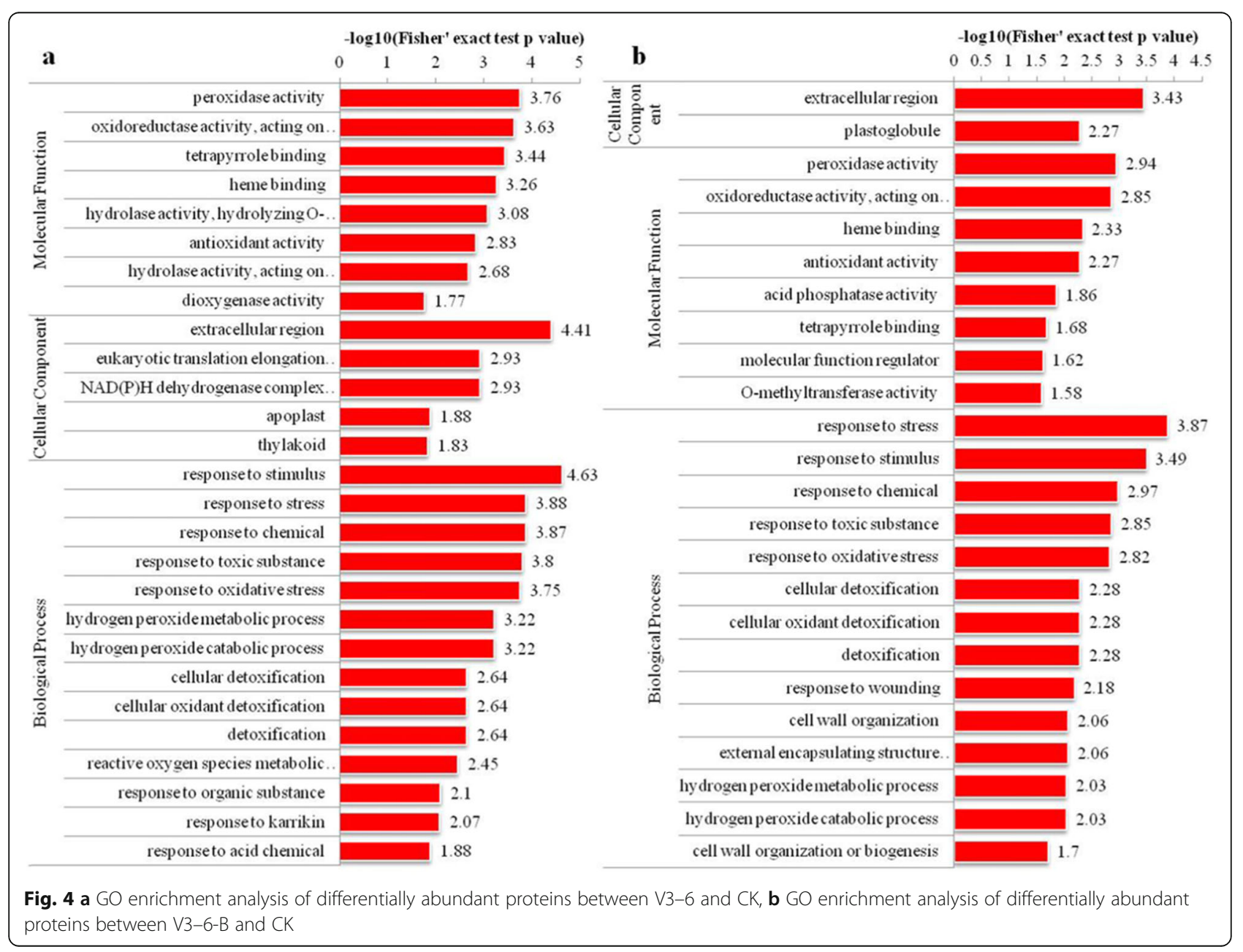




\section{Differentially abundant proteins involved in ROS scavenging system}

Proteomic data showed that proteins involved in ROS scavenging system such as peroxidase (A0A1D6E530, B4FBH0, B4FKV6, B4FVT1, COHHA6) were significantly up-accumulated in V3-6 treatment compared with CK. While, L-ascorbate peroxidase (A0A1D6QMU5), Glutathione peroxidase (C0P3R8) and APx3Peroxisomal Ascorbate Peroxidase (B6TM55) were down-accumulated in V3-6 treatment compared with CK. However, the peroxidases mentioned above were down-accumulated in V3-6-B treatment compared with V3-6. And L-ascorbate peroxidase (A0A1D6QMU5), Glutathione peroxidase (COP3R8) and APx3Peroxisomal Ascorbate Peroxidase (B6TM55) were up but not significantly accumulated in V3-6-B treatment compared with V3-6 (Table 1).

\section{Differentially abundant proteins involved in fatty acid metabolism}

Proteins that up-accumulated in V3-6-B and V3-6 compared with CK were strongly enriched in Linoleic acid metabolism and alpha- Linolenic acid metabolism, including Alpha-dioxygenase 1(H9BG22 and A0A1D6P493), Lipoxygenase (Q9LKL4). In addition, proteins involved in fatty acid metabolism such as Acyl carrier protein (B6UHG1, B6SJF5, B4FDG2), Diphosphonucleotide phosphatase1 (COPES7) and Triglyceride lipases (A0A1D6JA03) were up-accumulated in V3-6, but downaccumulated in V3-6-B. However, Fatty acid desaturase8 were down-regulated in V3-6 and V3-6-B (Table 2; Fig. 5).

\section{Differentially abundant proteins involved in proteins metabolism}

Compared with V3-6, the down-accumulated proteins of V3-6-B were enriched into 26 functional groups, of which molecular functions and biological processes accounted for 8 and $14 \mathrm{GO}$ terms, respectively. Interestingly, the most up-accumulated proteins were involved in proteins metabolism including "unfolded protein binding", "protein binding", "protein transporter activity", "protein disulfide isomerase activity", and "protein folding" (Table 3; Fig. 6).

\section{Discussion}

Plants are continuously exposed to environmental fluctuations, thus, they are facing great challenges to adapt themselves to variable environmental conditions, especially drought, waterlogging, cold, heat and other unfavorable conditions. To a certain extent, plant oxidative state could reflect the plant capability to resist biotic or abiotic stresses [33-35]. The generation and scavenging of ROS is one of plant defense mechanism to abiotic stresses. When plant is facing environmental stresses, ROS with high chemical activities are produced which could activate plant defense mechanism. However, excessive ROS would cause great damage to biological macromolecules such as proteins, nucleic acids and lipids, thus affecting their normal physiological and biochemical functions $[33,36]$. In addition, fatty acid metabolism plays an important role in plant defense by maintaining the integrity of the cell membranes. And, proteins metabolism plays an important role in plant defense by governing a series of plant defense activities $[37,38]$. Application of 6-BA and proteomic analysis enables us to obtain deeper insight into plant defense mechanism of summer maize to waterlogging. In this study, we found 6-BA improved waterlogged defense system by regulating a number of the proteins involved in ROS scavenging system, fatty acid and protein metabolism processes.

\section{ROS scavenging system activity}

Accumulation of excessive ROS is an anticipated common damage under stress conditions, which could also cause serious damages to organelles such as chloroplasts, mitochondria and plasma membranes [39]. Initially, ROS accumulation stimulated protective mechanisms by initiating signal cascades in vivo. However, when ROS are accumulated to a certain high level, they will attack proteins in various manners, leading to modification of proteins such as peroxidation of amino acid residues and oxidation of sulfur groups. As a result, the structure and function of proteins are changed, and ultimately resulting in distortion of cell structures, and acceleration of plant senescence. Previous studies have shown that ROS could cause membrane peroxidation injury along with a series of damages on plant growth, such as chlorophyll degradation, aging acceleration, and impaired photosynthesis [40-42]. Protective enzymes including SOD, POD and CAT as well as ascorbic acid, glutathione such antioxidants which play functions in scavenging ROS are employed to mitigate the oxidative damage and protect cells. Peroxidases participate in the reduction of ROS by catalyzing the redox reaction of $\mathrm{H}_{2} \mathrm{O}_{2}$ with various hydrogen donors [43]. Because there are many genes encoding peroxidases in plant cells, numerous types of peroxidase have important roles in plant stress resistance. For example, peroxidase 21 and peroxidase 42 played a significant role in cucumber plants response to waterlogging stress [44]. And, ascorbic acid peroxidase in the cytoplasm or combined with the cell wall played key roles in ascorbic acid-glutathione cycle, acting as a terminal oxidase. Ascorbic acid peroxidase, which was regulated by various signal molecules, could efficiently scavenge excess $\mathrm{H}_{2} \mathrm{O}_{2}[45,46]$. 
Table 1 Differentially abundant proteins involved in ROS scavenging system

\begin{tabular}{|c|c|c|c|c|c|c|c|c|c|c|c|c|}
\hline $\begin{array}{l}\text { Protein } \\
\text { accession }\end{array}$ & Protein description & Score & $\begin{array}{l}\text { Coverage } \\
{[\%]}\end{array}$ & Peptides & PSMs & $\begin{array}{l}\text { Unique } \\
\text { peptides }\end{array}$ & $\begin{array}{l}\text { V3- } \\
6 / C K \\
\text { Ratio }\end{array}$ & $\begin{array}{l}\text { V3-6/ } \\
\text { CK } \\
P \text { value }\end{array}$ & $\begin{array}{l}\text { V3- } \\
6-B / \\
\text { CK } \\
\text { Ratio }\end{array}$ & $\begin{array}{l}\text { V3-6-B/CK } \\
P \text { value }\end{array}$ & $\begin{array}{l}\text { V3- } \\
\text { 6-B/ } \\
\text { V3-6 } \\
\text { Ratio }\end{array}$ & $\begin{array}{l}\text { V3-6-B/ } \\
\text { V3-6 } \\
P \text { value }\end{array}$ \\
\hline A0A1D6E530 & $\begin{array}{l}\text { Peroxidase OS = Zea mays } \\
\text { GN = ZEAMMB73- } \\
\text { Zm00001d002899 }\end{array}$ & 160 & 47.2 & 9 & 15 & 9 & 1.57 & 0.0006 & 1.49 & 0.000737 & 0.95 & 0.115 \\
\hline A0A1D6HAE3 & $\begin{array}{l}\text { L-ascorbate peroxidase } \mathrm{S} \\
\text { chloroplastic/mitochondrial } \\
\text { OS = Zea mays GN = } \\
\text { ZEAMMB73_Zm00001d016802 }\end{array}$ & 288 & 37.2 & 16 & 40 & 13 & 1.15 & 0.00516 & 0.74 & 0.00000033 & 0.64 & 0.00000127 \\
\hline A0A1D613Z9 & $\begin{array}{l}\text { Putative cinnamyl alcohol } \\
\text { dehydrogenase } 6 \text { OS = Zea } \\
\text { mays GN = ZEAMMB73_- } \\
\text { Zm00001d020402 }\end{array}$ & 101 & 48.4 & 5 & 19 & 5 & 1.20 & 0.00048 & 1.23 & 0.000218 & 1.02 & 0.258 \\
\hline A0A1D6N0K3 & $\begin{array}{l}\text { Peroxidase OS = Zea mays } \\
\text { GN }=\text { ZEAMMB73_- } \\
\text { Zm00001d042022 }\end{array}$ & 79 & 25.2 & 8 & 15 & 8 & 1.28 & 0.0003 & 0.83 & 0.000856 & 0.65 & 0.0000788 \\
\hline A0A1D6QMU5 & $\begin{array}{l}\text { L-ascorbate peroxidase } 3 \\
\text { peroxisomal OS = Zea mays } \\
\text { GN = ZEAMMB73_- } \\
\text { Zm00001d053223 }\end{array}$ & 123 & 38.3 & 13 & 24 & 11 & 0.69 & 0.00014 & 0.78 & 0.000622 & 1.13 & 0.000801 \\
\hline A5H8G4 & $\begin{array}{l}\text { Peroxidase } 1 \text { OS = Zea mays } \\
\text { GN=PER1 }\end{array}$ & 131 & 34.6 & 10 & 23 & 8 & 1.22 & 0.00024 & 1.10 & 0.0147 & 0.90 & 0.00762 \\
\hline B4F7T9 & $\begin{array}{l}\text { Peroxidase OS = Zea mays } \\
\text { GN = ZEAMMB73- } \\
\text { Zm00001d023899 }\end{array}$ & 75 & 25.7 & 7 & 12 & 6 & 1.31 & 0.00068 & 1.15 & 0.024 & 0.88 & 0.0117 \\
\hline $\mathrm{B} 4 \mathrm{FBHO}$ & $\begin{array}{l}\text { Peroxidase OS = Zea mays } \\
\text { GN }=\text { ZEAMMB73- } \\
\text { Zm00001d024734 }\end{array}$ & 89 & 26.3 & 7 & 9 & 7 & 2.76 & $2.3 \mathrm{E}-07$ & 1.89 & $9.21 \mathrm{E}-07$ & 0.69 & 0.0000167 \\
\hline B4FKV6 & $\begin{array}{l}\text { Peroxidase OS = Zea mays } \\
\text { GN }=\text { ZEAMMB73 } \\
\text { Zm00001d014341 }\end{array}$ & 27 & 9.9 & 4 & 5 & 4 & 1.65 & 0.00014 & 0.86 & 0.0186 & 0.52 & 0.0000046 \\
\hline B4FVT1 & $\begin{array}{l}\text { Peroxidase OS = Zea mays } \\
\text { GN = ZEAMMB73- } \\
\text { Zm00001d037550 }\end{array}$ & 29 & 10.4 & 4 & 7 & 3 & 1.75 & 0.0012 & 1.01 & 0.547 & 0.58 & 0.0000226 \\
\hline B6TM55 & $\begin{array}{l}\text { APx1-Cytosolic Ascorbate } \\
\text { Peroxidase OS = Zea mays } \\
\text { GN = ZEAMMB73- } \\
\text { Zm00001d047757 }\end{array}$ & 152 & 61.6 & 13 & 40 & 3 & 0.84 & 0.00514 & 0.90 & 0.0461 & 1.07 & 0.024 \\
\hline $\mathrm{COHHAG}$ & $\begin{array}{l}\text { Peroxidase OS = Zea mays } \\
\text { GN }=\text { ZEAMMB73- } \\
\text { Zm00001d008173 }\end{array}$ & 8 & 2.6 & 1 & 1 & 1 & 2.50 & $8.5 \mathrm{E}-05$ & 1.72 & 0.00208 & 0.69 & 0.00354 \\
\hline COP3R8 & $\begin{array}{l}\text { Glutathione peroxidase OS = } \\
\text { Zea mays GN = ZEAMMB73_ } \\
\text { Zm00001d037079 }\end{array}$ & 65 & 35.2 & 7 & 19 & 7 & 0.78 & $2.4 \mathrm{E}-05$ & 0.85 & 0.000496 & 1.09 & 0.0039 \\
\hline C4J6E4 & $\begin{array}{l}\text { Peroxidase OS = Zea mays } \\
\text { GN = ZEAMMB73- } \\
\text { Zm00001d022456 }\end{array}$ & 39 & 34.6 & 4 & 7 & 4 & 0.94 & 0.264 & 0.67 & 0.00502 & 0.71 & 0.0136 \\
\hline K7VCN5 & $\begin{array}{l}\text { Peroxidase OS = Zea mays } \\
\text { GN = ZEAMMB73- } \\
\text { Zm00001d009373 }\end{array}$ & 146 & 34 & 7 & 7 & 7 & 1.44 & 0.00074 & 1.28 & 0.00106 & 0.89 & 0.0583 \\
\hline
\end{tabular}

This study showed that peroxidase (A0A1D6E530, B4FBH0, B4FKV6, B4FVT1, and C0HHA6) were upregulated in the V3-6 and down-regulated in V3-6-B compared with CK. Although, down-regulation of Lascorbate peroxidase (A0A1D6QMU5), Glutathione peroxidase (COP3R8) and APx3-Peroxisomal Ascorbate
Peroxidase (B6TM55) were observed in V3-6 compared with $\mathrm{CK}$, no significant up-regulation of these proteins were observed in V3-6-B compared with V3-6. However, the activity of SOD, POD and CAT were significantly decreased and the contents of MDA and $\mathrm{H}_{2} \mathrm{O}_{2}$ were increased greatly in V3-6, compared with CK. 
Table 2 Differentially abundant proteins involved in fatty acid metabolism

\begin{tabular}{|c|c|c|c|c|c|c|c|c|c|c|c|c|}
\hline $\begin{array}{l}\text { Protein } \\
\text { accession }\end{array}$ & Protein description & Score & $\begin{array}{l}\text { Coverage } \\
{[\%]}\end{array}$ & Peptides & PSMs & $\begin{array}{l}\text { Unique } \\
\text { peptides }\end{array}$ & $\begin{array}{l}\text { V3- } \\
6 / C K \\
\text { Ratio }\end{array}$ & $\begin{array}{l}\text { V3-6/ } \\
\text { CK } \\
P \text { value }\end{array}$ & $\begin{array}{l}\text { V3-6- } \\
\text { B/CK } \\
\text { Ratio }\end{array}$ & $\begin{array}{l}\text { V3-6-B/ } \\
\text { CK } \\
P \text { value }\end{array}$ & $\begin{array}{l}\text { V3-6- } \\
\text { B/N3- } \\
6 \\
\text { Ratio }\end{array}$ & $\begin{array}{l}\text { V3-6-B/ } \\
\text { V3-6 } \\
P \text { value }\end{array}$ \\
\hline A0A1D6G949 & $\begin{array}{l}\text { Protein FATTY ACID EXPORT } 3 \\
\text { chloroplastic OS = Zea mays } \\
\text { GN = ZEAMMB73_- } \\
\text { Zm00001d012504 }\end{array}$ & 69 & 14.6 & 4 & 5 & 1 & 1.22 & 0.041 & 0.93 & 0.621 & 0.77 & 0.192 \\
\hline A0A1D6J514 & $\begin{array}{l}\text { Fatty acid biosynthesis1 OS = } \\
\text { Zea mays GN = ZEAMMB73_- } \\
\text { Zm00001d025201 }\end{array}$ & 41 & 39.7 & 7 & 10 & 4 & 1.47 & 0.00066 & 1.34 & 0.00158 & 0.92 & 0.00964 \\
\hline A0A1D6JA03 & $\begin{array}{l}\text { Triglyceride lipases OS = Zea } \\
\text { mays GN = ZEAMMB73_- } \\
\text { Zm00001d025827 }\end{array}$ & 20 & 2.5 & 1 & 1 & 1 & 1.90 & 0.0115 & 0.72 & 0.139 & 0.38 & 0.000195 \\
\hline A0A1D6JQF2 & $\begin{array}{l}\text { Lipoxygenase OS = Zea mays } \\
\text { GN = ZEAMMB73- } \\
\text { Zm00001d027893 }\end{array}$ & 8 & 2.5 & 1 & 1 & 1 & 1.62 & 0.00736 & 0.90 & 0.314 & 0.56 & 0.00432 \\
\hline A0A1D6JZ55 & $\begin{array}{l}\text { Fatty acid desaturase8 OS = Zea } \\
\text { mays GN = ZEAMMB73__ } \\
\text { Zm00001d028742 }\end{array}$ & 14 & 3.8 & 2 & 2 & 2 & 0.80 & 0.00052 & 0.84 & 0.0041 & 1.05 & 0.245 \\
\hline A0A1D6P493 & $\begin{array}{l}\text { Alpha-dioxygenase } 1 \text { OS = Zea } \\
\text { mays GN = ZEAMMB73_- } \\
\text { Zm00001d046636 }\end{array}$ & 12 & 5.2 & 1 & 1 & 1 & 1.60 & 0.00476 & 1.87 & 0.001 & 1.17 & 0.111 \\
\hline B4FDG2 & $\begin{array}{l}\text { Acyl carrier protein OS = Zea } \\
\text { mays GN = ZEAMMB73_ } \\
\text { Zm00001d041701 }\end{array}$ & 14 & 17.1 & 2 & 6 & 2 & 1.94 & 0.00016 & 0.93 & 0.343 & 0.48 & 0.000521 \\
\hline B4FVE3 & $\begin{array}{l}\text { Farnesyl diphosphate synthase } 3 \\
\text { OS = Zea mays GN = } \\
\text { ZEAMMB73_Zm00001d043727 }\end{array}$ & 20 & 8.2 & 3 & 5 & 3 & 2.06 & $2.2 \mathrm{E}-05$ & 0.50 & 0.000117 & 0.24 & 0.00000415 \\
\hline B6SJF5 & $\begin{array}{l}\text { Acyl carrier protein OS = Zea } \\
\text { mays GN = ZEAMMB73_ } \\
\text { Zm00001d032019 }\end{array}$ & 7 & 8.4 & 1 & 1 & 1 & 1.96 & $2.4 \mathrm{E}-06$ & 0.72 & 0.000344 & 0.37 & 0.0000025 \\
\hline B6UHG1 & $\begin{array}{l}\text { Acyl carrier protein OS = Zea } \\
\text { mays GN = ZEAMMB73_ } \\
\text { Zm00001d019186 }\end{array}$ & 7 & 6.8 & 1 & 3 & 1 & 1.37 & 0.00112 & 0.86 & 0.0269 & 0.63 & 0.000222 \\
\hline COPES7 & $\begin{array}{l}\text { Diphosphonucleotide } \\
\text { phosphatase1 OS = Zea mays } \\
\text { GN = ZEAMMB73- } \\
\text { Zm00001d023698 }\end{array}$ & 186 & 30.7 & 10 & 21 & 10 & 1.34 & $4.5 \mathrm{E}-06$ & 0.87 & 0.00128 & 0.65 & 0.00000418 \\
\hline H9BG22 & $\begin{array}{l}\text { Alpha-dioxygenase } 1 \text { OS = Zea } \\
\text { mays GN = ZEAMMB73_ } \\
\text { Zm00001d035854 }\end{array}$ & 96 & 21 & 11 & 16 & 11 & 1.56 & $5.9 \mathrm{E}-05$ & 1.65 & 0.000024 & 1.06 & 0.0474 \\
\hline Q9LKL4 & $\begin{array}{l}\text { Lipoxygenase OS = Zea mays } \\
\text { GN = LOX }\end{array}$ & 188 & 26.5 & 20 & 25 & 20 & 1.93 & 0.00822 & 1.65 & 0.0141 & 0.85 & 0.000176 \\
\hline
\end{tabular}

And, these enzymes activities were increased and the MDA, $\mathrm{H}_{2} \mathrm{O}_{2}$ contents were effectively reduced by application of 6-BA, compared to those of waterlogging treatment. These results suggested that there may exist some additional complex mechanisms to regulate the ROS scavenging system activity besides regulating the protein expression level.

\section{Fatty acid metabolism}

Maintenance of membrane integrity under stress broadly reflected intrinsic tolerance [47]. Regarding fatty acid metabolism, alpha-dioxygenase 1 (A0A1D6P493 and H9BG22) and cytochrome P450 CYP74A19
(A0A096PQR7) were upregulated in both the V3-6 and $\mathrm{V} 3-6-\mathrm{B}$ treatments, and increased more in $\mathrm{V3}-6-\mathrm{B}$ than in V3-6. In addition, lipoxygenase (Q9LKL4) also showed an increase in both V3-6 and V3-6-B. However, the magnitude of the increase was significantly higher in V3-6 than in V3-6-B, while, lipoxygenase (A0A1D6JQF2) and acyl carrier protein (B4FDG2 and B6SJF5) were up-regulated in V3-6 and down-regulated in V3-6-B. Plant alpha-dioxygenases convert fatty acids to 2-hydroperoxy products which plays an important role in plant signaling pathways [48]. Cytochrome P450s exist ubiquitously in all organisms playing important roles in many biological processes. For example, 


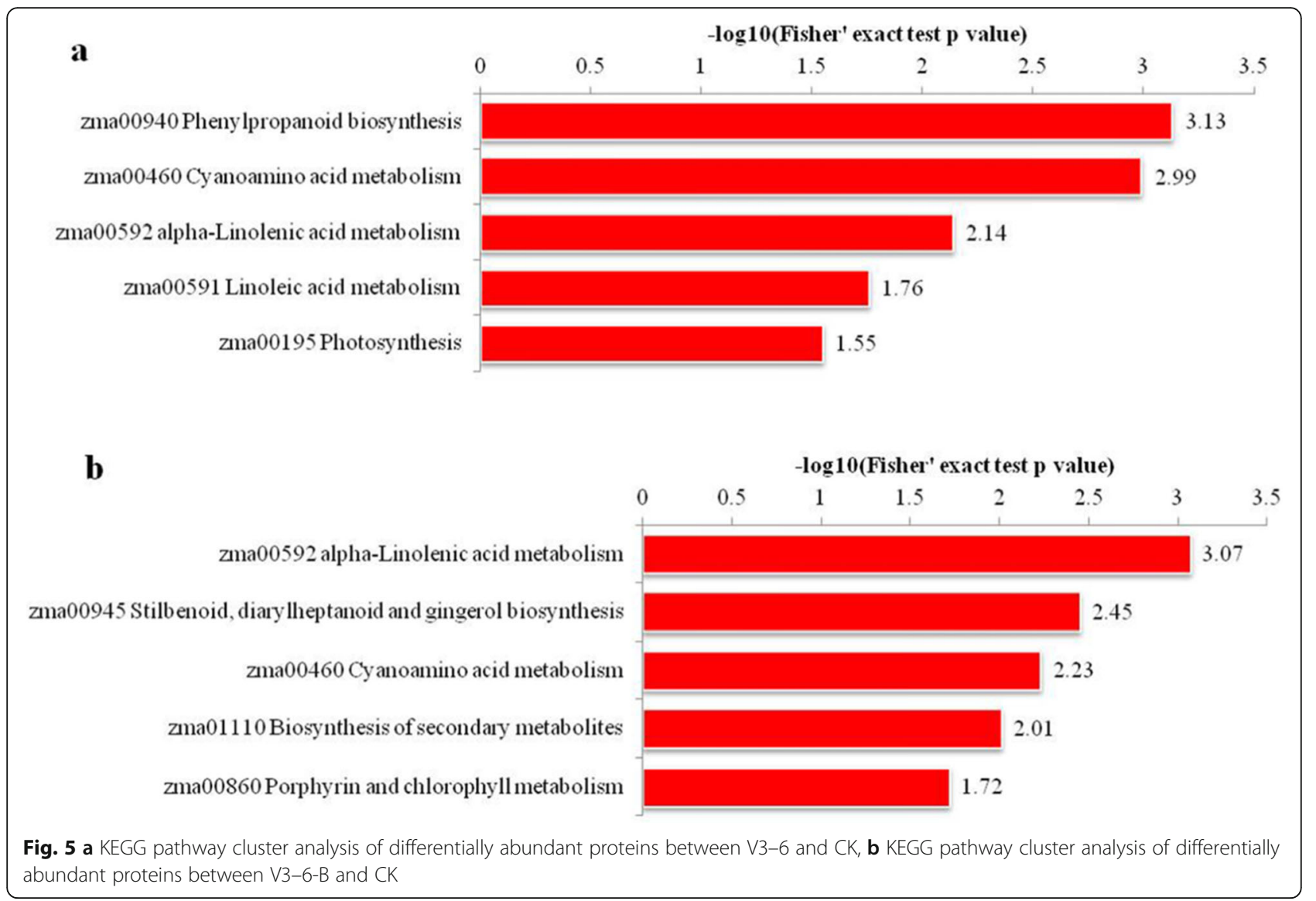

Cytochrome P450 A2 plays a key role in signal and defense reactions in higher plants [49]. The upregulation of these two enzymes in V3-6 and V3-6-B treatments revealed that plant employed a positive response mechanism to address waterlogging stress. Acyl carrier protein (B4FDG2 and B6SJF5), lipoxygenase (A0A1D6JQF2 and Q9LKL4) and cytochrome P450 CYP74A19 (A0A096PQR7) were enriched in fatty acid biosynthesis pathway. The protein-protein network of interactions showed that lipoxygenase (A0A1D6JQF2) might bind with cytochrome P450 CYP74A19 (A0A096PQR7) to perform their functions. In addition, lipoxygenase (A0A1D6JQF2 and Q9LKL4) and cytochrome P450 CYP74A19 (A0A096PQR7) were also enriched in alpha-Linolenic acid metabolism. Furthermore, lipoxygenase (A0A1D6JQF2 and Q9LKL4) had interactions with alpha-dioxygenase 1 (A0A1D6P493 and H9BG22), which also played important roles in alphaLinolenic acid metabolism (Fig. 7). These results showed that lipoxygenase (A0A1D6JQF2 and Q9LKL4) played a central role in both alpha-Linolenic acid metabolism and fatty acid biosynthesis pathway.

Alpha-linolenic acid, an important precursor of the cell signaling molecule jasmonic acid, could induce jasmonic acid accumulation. Also, alpha-linolenic acid appears to be necessary for biomembrane integrity and function, which plays a key role in protecting plants from environmental stress damage. Plant resistance to cold is weakened with a decrease in unsaturated acids, as shown in Arabidopsis thaliana by mutating the chloroplast sn - 2-palmitoyl desaturase and $\Delta 12$-desaturase genes [50, 51]. Increasing the expression of fatty acid-desaturating genes in plants can increase the production of alpha-linolenic acid [52, 53]. As the membrane components, particularly of phospholipids, Linoleic and alpha-Linolenic acids play key roles in maintaining membrane integrity. In addition, they are also substrates for lipoxygenase (LOX). Therefore, LOX could do destructive damages to cell membranes and other cell components [54]. An increase in lipoxygenase always accompanied damage of unsaturated fatty acids, as lipoxygenase could oxidize unsaturated fatty acids and incur lipid peroxidation as well as a series of secondary reactions [55]. In addition, lipoxygenase reactions are also the sources of ROS [56]. Excessive LOX and ROS could induce lipid peroxidation, loss of membrane integrity which has been proved in a wide range of plants such as wheat, bean, cotton, maize and so forth. Interestingly, a close association between LOX and ROS has also been established [54]. 
Table 3 Differentially abundant proteins involved in proteins metabolism

\begin{tabular}{|c|c|c|c|c|c|c|c|c|c|c|c|c|}
\hline $\begin{array}{l}\text { Protein } \\
\text { accession }\end{array}$ & Protein description & Score & $\begin{array}{l}\text { Coverage } \\
{[\%]}\end{array}$ & Peptides & PSMs & $\begin{array}{l}\text { Unique } \\
\text { peptides }\end{array}$ & $\begin{array}{l}\text { V3- } \\
6 / C K \\
\text { Ratio }\end{array}$ & $\begin{array}{l}\text { V3-6/ } \\
\text { CK } P \\
\text { value }\end{array}$ & $\begin{array}{l}\text { V3- } \\
6-B / C K \\
\text { Ratio }\end{array}$ & $\begin{array}{l}\text { V3-6- } \\
\text { B/CK } \\
P \text { value }\end{array}$ & $\begin{array}{l}\text { V3-6-B/ } \\
\text { V3-6 } \\
\text { Ratio }\end{array}$ & $\begin{array}{l}\text { V3-6-B/ } \\
\text { V3-6 } \\
P \text { value }\end{array}$ \\
\hline A0A1D6E942 & $\begin{array}{l}30 S \text { ribosomal protein } 3 \\
\text { chloroplastic OS = Zea mays } \\
\text { GN = ZEAMMB73_- } \\
\text { Zm00001d003411 }\end{array}$ & 19 & 16 & 3 & 6 & 3 & 1.51 & $5.8 \mathrm{E}-05$ & 0.86 & 0.0026 & 0.57 & 0.00000432 \\
\hline A0A1D6ES19 & $\begin{array}{l}\text { Heat shock protein } 90-2 \\
\text { OS }=\text { Zea mays } \\
\text { GN = ZEAMMB73_- } \\
\text { Zm00001d006008 }\end{array}$ & 16 & 36.4 & 21 & 27 & 2 & 1.63 & 0.00058 & 0.98 & 0.765 & 0.60 & 0.000603 \\
\hline A0A1D6GG02 & $\begin{array}{l}\text { DnaJ protein OS = Zea mays } \\
\text { GN = ZEAMMB73_- } \\
\text { Zm00001d013111 }\end{array}$ & 12 & 30.7 & 12 & 17 & 2 & 1.13 & 0.0477 & 0.71 & 0.00432 & 0.63 & 0.00202 \\
\hline A0A1D6GK64 & $\begin{array}{l}\text { Heat shock } 70 \mathrm{kDa} \text { protein } 6 \\
\text { chloroplastic OS = Zea mays } \\
\text { GN = ZEAMMB73- } \\
\text { Zm00001d013507 }\end{array}$ & 44 & 51.2 & 36 & 125 & 2 & 1.17 & 0.0416 & 0.72 & 0.0186 & 0.62 & 0.00404 \\
\hline A0A1D6GTD7 & $\begin{array}{l}26 \mathrm{~S} \text { proteasome non-ATPase } \\
\text { regulatory subunit } 13 \text { homo } \\
\text { log } \mathrm{A} \text { OS }=\text { Zea mays GN = } \\
\text { ZEAMMB73_Zm00001d014436 }\end{array}$ & 49 & 20.9 & 6 & 6 & 3 & 1.51 & 0.0122 & 1.02 & 0.895 & 0.67 & 0.0311 \\
\hline A0A1D6GWT5 & $\begin{array}{l}\text { Calcium-binding EF hand } \\
\text { family protein OS = Zea mays } \\
\text { GN = ZEAMMB73_- } \\
\text { Zm00001d014862 }\end{array}$ & 16 & 1.7 & 2 & 2 & 2 & 1.31 & 0.0191 & 0.85 & 0.0303 & 0.65 & 0.00178 \\
\hline A0A1D6GZM9 & $\begin{array}{l}\text { RING/FYVE/PHD zinc finger } \\
\text { superfamily protein OS = Zea } \\
\text { mays GN = ZEAMMB73_- } \\
\text { Zm00001d015152 }\end{array}$ & 6 & 10.8 & 1 & 1 & 1 & 2.35 & 0.00596 & 0.63 & 0.00938 & 0.27 & 0.00328 \\
\hline A0A1D6GZY9 & $\begin{array}{l}\text { Cleavage and polyadenylation } \\
\text { specificity factor (CPSF) A } \\
\text { subunit protein OS = Zea } \\
\text { mays GN = ZEAMMB73_- } \\
\text { Zm00001d015208 }\end{array}$ & 65 & 5.6 & 7 & 9 & 7 & 1.43 & 0.00056 & 0.82 & 0.0192 & 0.57 & 0.00026 \\
\hline A0A1D6H4K9 & $\begin{array}{l}\text { Nucleic acid-binding OB-fold- } \\
\text { like protein OS = Zea mays } \\
\text { GN = ZEAMMB73- } \\
\text { Zm00001d015917 }\end{array}$ & 16 & 16.2 & 2 & 2 & 2 & 1.57 & $1.8 \mathrm{E}-05$ & 1.10 & 0.0534 & 0.71 & 0.000604 \\
\hline A0A1D6H5B6 & $\begin{array}{l}50 S \text { ribosomal protein L21 } \\
\text { chloroplastic OS = Zea mays } \\
\text { GN = ZEAMMB73_- } \\
\text { Zm00001d016072 }\end{array}$ & 49 & 34 & 4 & 7 & 4 & 1.60 & 0.00016 & 0.82 & 0.0385 & 0.51 & 0.000382 \\
\hline A0A1D6HCE3 & $\begin{array}{l}26 \mathrm{~S} \text { proteasome non-ATPase } \\
\text { regulatory subunit } 6 \text {-like pro } \\
\text { tein OS = Zea mays GN }= \\
\text { ZEAMMB73_Zm00001d017117 }\end{array}$ & 36 & 11.4 & 4 & 7 & 2 & 1.80 & $1.8 \mathrm{E}-06$ & 0.93 & 0.182 & 0.52 & 0.000125 \\
\hline A0A1D6HG23 & $\begin{array}{l}\text { Uncharacterized protein OS = } \\
\text { Zea mays GN = ZEAMMB73_- } \\
\text { Zm00001d017621 }\end{array}$ & 61 & 25.5 & 4 & 10 & 4 & 1.24 & 0.00136 & 0.73 & 0.00000299 & 0.59 & 0.0000366 \\
\hline A0A1D6HT76 & $\begin{array}{l}\text { Protein containing PDZ } \\
\text { domain a K-box domain and } \\
\text { a TPR region OS = Zea mays } \\
\text { GN = ZEAMMB73_- } \\
\text { Zm00001d018901 }\end{array}$ & 159 & 37.6 & 9 & 21 & 9 & 1.62 & $1.1 \mathrm{E}-06$ & 0.65 & 0.000341 & 0.40 & 0.00164 \\
\hline A0A1D6 $\| 13$ & $\begin{array}{l}\text { Calcium-dependent lipid- } \\
\text { binding (CaLB domain) } \\
\text { family protein OS = Zea } \\
\text { mays GN = ZEAMMB73_- } \\
\text { Zm00001d021952 }\end{array}$ & 27 & 6.5 & 2 & 2 & 1 & 1.22 & 0.0366 & 0.80 & 0.0167 & 0.66 & 0.00254 \\
\hline A0A1D6JX93 & $\begin{array}{l}\text { Peroxisomal nicotinamide } \\
\text { adenine dinucleotide carrier } \\
\text { OS = Zea mays GN = ZEAMMB73_ } \\
\text { Zm00001d028542 }\end{array}$ & 32 & 14.6 & 4 & 5 & 4 & 1.59 & 0.00092 & 1.00 & 0.976 & 0.63 & 0.000185 \\
\hline A0A1D6KA58 & 265 proteasome non-ATPase & 24 & 15.6 & 5 & 5 & 2 & 1.52 & $7.5 \mathrm{E}-05$ & 1.23 & 0.00482 & 0.81 & 0.00676 \\
\hline
\end{tabular}


Table 3 Differentially abundant proteins involved in proteins metabolism (Continued)

\begin{tabular}{|c|c|c|c|c|c|c|c|c|c|c|c|c|}
\hline $\begin{array}{l}\text { Protein } \\
\text { accession }\end{array}$ & Protein description & Score & $\begin{array}{l}\text { Coverage } \\
{[\%]}\end{array}$ & Peptides & PSMs & $\begin{array}{l}\text { Unique } \\
\text { peptides }\end{array}$ & $\begin{array}{l}\text { V3- } \\
6 / C K \\
\text { Ratio }\end{array}$ & $\begin{array}{l}\text { V3-6/ } \\
\text { CK } P \\
\text { value }\end{array}$ & $\begin{array}{l}\text { V3- } \\
6-\mathrm{B} / \mathrm{CK} \\
\text { Ratio }\end{array}$ & $\begin{array}{l}\text { V3-6- } \\
\text { B/CK } \\
P \text { value }\end{array}$ & $\begin{array}{l}\text { V3-6-B/ } \\
\text { V3-6 } \\
\text { Ratio }\end{array}$ & $\begin{array}{l}\text { V3-6-B/ } \\
\text { V3-6 } \\
P \text { value }\end{array}$ \\
\hline & $\begin{array}{l}\text { regulatory subunit } 13 \text { homolog A } \\
\text { OS = Zea mays GN = ZEAMMB73_ } \\
\text { Zm00001d030126 }\end{array}$ & & & & & & & & & & & \\
\hline A0A1D6KE00 & $\begin{array}{l}\text { Thaumatin-like protein OS = Zea } \\
\text { mays GN = ZEAMMB73_ } \\
\text { Zm00001d030694 }\end{array}$ & 204 & 21.5 & 18 & 25 & 9 & 1.29 & 0.00458 & 0.80 & 0.157 & 0.62 & 0.0219 \\
\hline A0A1D6KJP4 & $\begin{array}{l}\text { Peptidylprolyl isomerase OS = } \\
\text { Zea mays GN = ZEAMMB73_- } \\
\text { Zm00001d031569 }\end{array}$ & 124 & 27.6 & 13 & 16 & 13 & 1.48 & $9.2 \mathrm{E}-07$ & 0.82 & 0.00128 & 0.55 & 0.0000189 \\
\hline A0A1D6KSJ1 & $\begin{array}{l}\text { Putative prefoldin subunit } 5 \\
\text { OS = Zea mays GN = ZEAMMB73 } \\
\text { Zm00001d032649 }\end{array}$ & 10 & 13.8 & 1 & 1 & 1 & 1.65 & 0.00494 & 1.06 & 0.598 & 0.64 & 0.00206 \\
\hline A0A1D6L6U7 & $\begin{array}{l}\text { NmrA-like negative } \\
\text { transcriptional regulator family } \\
\text { protein OS = Zea mays GN = } \\
\text { ZEAMMB73_Zm00001d034357 }\end{array}$ & 23 & 9.2 & 2 & 2 & 2 & 0.48 & $2.5 \mathrm{E}-08$ & 0.53 & 0.0111 & 1.12 & 0.248 \\
\hline A0A1D6LBS9 & $\begin{array}{l}\text { Protein prenylyltransferase } \\
\text { superfamily protein OS = Zea } \\
\text { mays GN = ZEAMMB73_- } \\
\text { Zm00001d034833 }\end{array}$ & 17 & 11.2 & 2 & 3 & 2 & 1.45 & 0.00226 & 0.68 & 0.0489 & 0.47 & 0.00486 \\
\hline A0A1D6LIX0 & $\begin{array}{l}\text { Protein kinase superfamily } \\
\text { protein with octicosapeptide/ } \\
\text { Phox/Bem1p domain OS = Zea } \\
\text { mays GN = ZEAMMB73_- } \\
\text { Zm00001d035817 }\end{array}$ & 59 & 8.1 & 8 & 9 & 6 & 1.33 & 0.00606 & 0.82 & 0.0307 & 0.62 & 0.0012 \\
\hline A0A1D6M323 & $\begin{array}{l}\text { Ribosomal protein OS = Zea } \\
\text { mays GN = ZEAMMB73_ } \\
\text { Zm00001d038084 }\end{array}$ & 236 & 42.3 & 15 & 35 & 14 & 2.01 & $1.7 \mathrm{E}-07$ & 0.69 & 0.0059 & 0.34 & 0.00000192 \\
\hline A0A1D6MBB0 & $\begin{array}{l}\text { Nucleosome assembly protein } 1 \\
\text { OS }=\text { Zea mays GN = ZEAMMB73_ } \\
\text { Zm00001d038851 }\end{array}$ & 25 & 7.5 & 2 & 3 & 1 & 1.89 & $2.9 \mathrm{E}-07$ & 0.90 & 0.205 & 0.48 & 0.00578 \\
\hline A0A1D6MDC0 & $\begin{array}{l}\text { Protein SUPPRESSOR OF PHYA- } \\
1051 \text { OS }=\text { Zea mays GN = } \\
\text { ZEAMMB73_Zm00001d039072 }\end{array}$ & -2 & 0.7 & 1 & 1 & 1 & 1.40 & 0.224 & 0.82 & 0.553 & 0.59 & 0.0255 \\
\hline A0A1D6MUF1 & $\begin{array}{l}\text { Heat shock } 70 \mathrm{kDa} \text { protein } 6 \\
\text { chloroplastic OS = Zea mays } \\
\text { GN = ZEAMMB73_- } \\
\text { Zm00001d041119 }\end{array}$ & 323 & 55 & 35 & 98 & 7 & 1.08 & 0.0124 & 0.69 & 0.0000396 & 0.64 & 0.0000363 \\
\hline A0A1D6MWV7 & $\begin{array}{l}\text { Importin subunit beta-1 OS = } \\
\text { Zea mays GN = ZEAMMB73 } \\
\text { Zm00001d041556 }\end{array}$ & 23 & 15.8 & 10 & 14 & 2 & 1.45 & 0.0107 & 0.78 & 0.0136 & 0.54 & 0.00206 \\
\hline A0A1D6NQK6 & $\begin{array}{l}\text { SMAD/FHA domain-containing } \\
\text { protein OS = Zea mays GN = } \\
\text { ZEAMMB73_Zm00001d044666 }\end{array}$ & 80 & 17.9 & 3 & 4 & 3 & 1.33 & 0.00962 & 0.73 & 0.00666 & 0.55 & 0.000518 \\
\hline A0A1D6PIZ9 & $\begin{array}{l}\text { Tubulin-tyrosine ligase OS = } \\
\text { Zea mays GN = ZEAMMB73_ } \\
\text { Zm00001d048282 }\end{array}$ & 30 & 5.1 & 4 & 5 & 4 & 1.02 & 0.853 & 0.68 & 0.0255 & 0.66 & 0.0168 \\
\hline A0A1D6QK75 & $\begin{array}{l}\text { Heat shock protein } 90-5 \\
\text { chloroplastic OS = Zea mays } \\
\text { GN = ZEAMMB73- } \\
\text { Zm00001d052809 }\end{array}$ & 34 & 11.9 & 8 & 10 & 3 & 1.40 & 0.0166 & 0.83 & 0.13 & 0.59 & 0.00038 \\
\hline $\mathrm{B} 4 \mathrm{~F} 8 \mathrm{Q} 2$ & $\begin{array}{l}\text { Uncharacterized protein OS = } \\
\text { Zea mays }\end{array}$ & 42 & 40.6 & 25 & 55 & 2 & 1.28 & 0.00678 & 0.62 & 0.00126 & 0.48 & 0.00038 \\
\hline B4FCK9 & 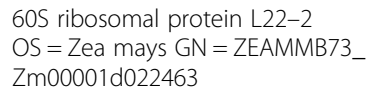 & 57 & 46.9 & 5 & 8 & 4 & 1.62 & 0.00148 & 0.89 & 0.196 & 0.55 & 0.000221 \\
\hline B4FI21 & $\begin{array}{l}\text { ARM repeat superfamily protein } \\
\text { OS = Zea mays GN = ZEAMMB73 } \\
\text { Zm00001d053003 }\end{array}$ & 161 & 36.5 & 13 & 17 & 3 & 1.10 & 0.1 & 0.64 & 0.000498 & 0.58 & 0.000156 \\
\hline B4FKB3 & $50 S$ ribosomal protein $\mathrm{L31} \mathrm{OS}=$ & 10 & 8.5 & 1 & 5 & 1 & 1.43 & 0.00054 & 0.78 & 0.00418 & 0.55 & 0.0000366 \\
\hline
\end{tabular}


Table 3 Differentially abundant proteins involved in proteins metabolism (Continued)

\begin{tabular}{|c|c|c|c|c|c|c|c|c|c|c|c|c|}
\hline $\begin{array}{l}\text { Protein } \\
\text { accession }\end{array}$ & Protein description & Score & $\begin{array}{l}\text { Coverage } \\
{[\%]}\end{array}$ & Peptides & PSMs & $\begin{array}{l}\text { Unique } \\
\text { peptides }\end{array}$ & $\begin{array}{l}\text { V3- } \\
\text { 6/CK } \\
\text { Ratio }\end{array}$ & $\begin{array}{l}\text { V3-6/ } \\
\text { CK } P \\
\text { value }\end{array}$ & $\begin{array}{l}\text { V3- } \\
6-\mathrm{B} / \mathrm{CK} \\
\text { Ratio }\end{array}$ & $\begin{array}{l}\text { V3-6- } \\
\text { B/CK } \\
P \text { value }\end{array}$ & $\begin{array}{l}\text { V3-6-B/ } \\
\text { V3-6 } \\
\text { Ratio }\end{array}$ & $\begin{array}{l}\text { V3-6-B/ } \\
\text { V3-6 } \\
P \text { value }\end{array}$ \\
\hline & $\begin{array}{l}\text { Zea mays GN }=\text { ZEAMMB73 } \\
\text { Zm00001d044130 }\end{array}$ & & & & & & & & & & & \\
\hline B4FNC9 & $\begin{array}{l}\text { Small ubiquitin-related modifier } \\
\text { OS = Zea mays GN=SUMO1a }\end{array}$ & 77 & 48.5 & 6 & 9 & 6 & 1.30 & 0.00172 & 0.74 & 0.00122 & 0.57 & 0.000018 \\
\hline B4FNT1 & $\begin{array}{l}\text { Elongation factor 1-beta OS = } \\
\text { Zea mays GN = ZEAMMB73 } \\
\text { Zm00001d022134 }\end{array}$ & 48 & 22.3 & 4 & 7 & 4 & 1.65 & 0.00034 & 0.96 & 0.378 & 0.58 & 0.000083 \\
\hline B4FT63 & $\begin{array}{l}\text { Genomes uncoupled4-like } \\
\text { protein OS }=\text { Zea mays GN }= \\
\text { ZEAMMB73_Zm00001d023427 }\end{array}$ & 13 & 7.4 & 2 & 2 & 2 & 1.52 & $4 \mathrm{E}-06$ & 0.69 & 0.0212 & 0.45 & 0.000141 \\
\hline B4FT80 & $\begin{array}{l}\text { Nucleic acid-binding OB-fold-like } \\
\text { protein OS = Zea mays GN = } \\
\text { ZEAMMB73_Zm00001d045607 }\end{array}$ & 8 & 8.4 & 1 & 1 & 1 & 1.60 & 0.00168 & 0.79 & 0.083 & 0.49 & 0.0028 \\
\hline B4FUA8 & $\begin{array}{l}\text { Calreticulin-3 OS = Zea mays } \\
\text { GN = ZEAMMB73- } \\
\text { Zm00001d012170 }\end{array}$ & 85 & 30.6 & 11 & 15 & 10 & 1.21 & 0.00336 & 0.68 & 0.0000216 & 0.56 & 0.0000245 \\
\hline B4FUZ5 & $\begin{array}{l}30 S \text { ribosomal protein } \mathrm{S1} \text { OS }= \\
\text { Zea mays GN = ZEAMMB73_- } \\
\text { Zm00001d047581 }\end{array}$ & 72 & 26.4 & 8 & 13 & 8 & 1.57 & $1.7 \mathrm{E}-05$ & 1.00 & 0.919 & 0.64 & 0.000465 \\
\hline $\mathrm{B} 4 \mathrm{FVI} 4$ & $\begin{array}{l}60 S \text { acidic ribosomal protein P2- } \\
5 \text { OS = Zea mays GN = } \\
\text { ZEAMMB73_Zm00001d026578 }\end{array}$ & 8 & 9 & 1 & 1 & 1 & 2.14 & 0.00022 & 1.38 & 0.000382 & 0.65 & 0.00128 \\
\hline B6SLK4 & $\begin{array}{l}\text { Elongation factor 1-beta OS = } \\
\text { Zea mays GN = ZEAMMB73 } \\
\text { Zm00001d022513 }\end{array}$ & 100 & 38.8 & 8 & 18 & 8 & 2.01 & $1.9 \mathrm{E}-07$ & 0.65 & 0.000197 & 0.32 & 0.00000202 \\
\hline B6T9G1 & $\begin{array}{l}26 S \text { proteasome non-ATPase } \\
\text { regulatory subunit } 6 \text { OS = Zea } \\
\text { mays GN = ZEAMMB73_ } \\
\text { Zm00001d003257 }\end{array}$ & 24 & 8.8 & 3 & 3 & 1 & 1.71 & 0.00012 & 0.80 & 0.00512 & 0.47 & 0.0000153 \\
\hline B6TBM1 & $\begin{array}{l}\text { Alpha-soluble NSF attachment } \\
\text { protein OS = Zea mays GN = } \\
\text { ZEAMMB73_Zm00001d049982 }\end{array}$ & 119 & 39.1 & 9 & 10 & 9 & 1.41 & 0.00308 & 0.78 & 0.000221 & 0.55 & 0.000385 \\
\hline B6TDB5 & $\begin{array}{l}17.4 \mathrm{kDa} \text { class I heat shock } \\
\text { protein } 3 \text { OS = Zea mays GN = } \\
\text { ZEAMMB73_Zm00001d028555 }\end{array}$ & 33 & 18.4 & 3 & 5 & 3 & 0.56 & $4.2 \mathrm{E}-06$ & 0.64 & 0.00132 & 1.14 & 0.0887 \\
\hline B6TRV8 & $\begin{array}{l}\text { ARM repeat superfamily protein } \\
\text { OS = Zea mays GN = ZEAMMB73_ } \\
\text { Zm00001d004676 }\end{array}$ & 13 & 28.5 & 11 & 15 & 1 & 1.15 & 0.0159 & 0.70 & 0.000737 & 0.61 & 0.000198 \\
\hline B6TT66 & $\begin{array}{l}\text { Ribosome-like protein OS = Zea } \\
\text { mays GN = ZEAMMB73_ } \\
\text { Zm00001d015628 }\end{array}$ & 41 & 28.1 & 4 & 6 & 3 & 1.39 & 0.0119 & 0.88 & 0.203 & 0.64 & 0.00216 \\
\hline $\mathrm{B} 6 \mathrm{U} 284$ & $\begin{array}{l}\text { 14-3-3-like protein OS }=\text { Zea } \\
\text { mays GN = ZEAMMB73_ } \\
\text { Zm00001d050375 }\end{array}$ & 48 & 43 & 10 & 13 & 3 & 1.84 & 0.00022 & 1.03 & 0.611 & 0.56 & 0.00082 \\
\hline B6U581 & $\begin{array}{l}\text { Ribosome-like protein OS = Zea } \\
\text { mays GN = ZEAMMB73_ } \\
\text { Zm00001d053713 }\end{array}$ & 57 & 42.4 & 6 & 10 & 5 & 1.35 & 0.00298 & 0.78 & 0.00218 & 0.58 & 0.00046 \\
\hline B6UG10 & $\begin{array}{l}40 S \text { ribosomal protein S3a OS = } \\
\text { Zea mays GN = ZEAMMB73_ } \\
\text { Zm00001d048157 }\end{array}$ & 85 & 35.8 & 10 & 18 & 10 & 1.34 & 0.0001 & 0.76 & 0.000324 & 0.57 & 0.0000355 \\
\hline B6UIC1 & $\begin{array}{l}50 S \text { ribosomal protein L12-1 } \\
\text { OS = Zea mays GN = ZEAMMB73 } \\
\text { Zm00001d043972 }\end{array}$ & 181 & 56.3 & 10 & 43 & 3 & 1.33 & 0.00082 & 0.82 & 0.00558 & 0.62 & 0.0000351 \\
\hline COHF19 & $\begin{array}{l}\text { Nucleosome assembly protein } 1 \\
\text { OS }=\text { Zea mays GN = ZEAMMB73 } \\
\text { Zm00001d045352 }\end{array}$ & 7 & 10 & 2 & 2 & 1 & 1.72 & $4.2 \mathrm{E}-05$ & 0.84 & 0.0165 & 0.49 & 0.000622 \\
\hline COP531 & $\begin{array}{l}26 \mathrm{~S} \text { proteasome non-ATPase } \\
\text { regulatory subunit } 3 \text { homolog A } \\
\text { OS = Zea mays GN = ZEAMMB73_ }\end{array}$ & 96 & 21.8 & 9 & 10 & 9 & 1.21 & 0.0251 & 0.72 & 0.00000138 & 0.60 & 0.0000803 \\
\hline
\end{tabular}


Table 3 Differentially abundant proteins involved in proteins metabolism (Continued)

\begin{tabular}{|c|c|c|c|c|c|c|c|c|c|c|c|c|}
\hline $\begin{array}{l}\text { Protein } \\
\text { accession }\end{array}$ & Protein description & Score & $\begin{array}{l}\text { Coverage } \\
{[\%]}\end{array}$ & Peptides & PSMs & $\begin{array}{l}\text { Unique } \\
\text { peptides }\end{array}$ & $\begin{array}{l}\text { V3- } \\
6 / C K \\
\text { Ratio }\end{array}$ & $\begin{array}{l}\text { V3-6/ } \\
\text { CK } P \\
\text { value }\end{array}$ & $\begin{array}{l}\text { V3- } \\
6-\mathrm{B} / \mathrm{CK} \\
\text { Ratio }\end{array}$ & $\begin{array}{l}\text { V3-6- } \\
\text { B/CK } \\
P \text { value }\end{array}$ & $\begin{array}{l}\text { V3-6-B/ } \\
\text { V3-6 } \\
\text { Ratio }\end{array}$ & $\begin{array}{l}\text { V3-6-B/ } \\
\text { V3-6 } \\
P \text { value }\end{array}$ \\
\hline & Zm00001d053232 & & & & & & & & & & & \\
\hline COPDG3 & $\begin{array}{l}\text { Heat shock protein 90-6 } \\
\text { mitochondrial OS = Zea mays } \\
\text { GN =ZEAMMB73_- } \\
\text { Zm00001d041719 }\end{array}$ & 162 & 20.8 & 13 & 16 & 8 & 1.45 & 0.00086 & 0.73 & 0.0189 & 0.51 & 0.0016 \\
\hline C4JBB8 & $\begin{array}{l}\text { Heat shock } 70 \mathrm{kDa} \text { protein } 9 \\
\text { mitochondrial OS = Zea mays } \\
\mathrm{GN}=\text { ZEAMMB73_- } \\
\text { Zm00001d006036 }\end{array}$ & 64 & 22.3 & 14 & 19 & 9 & 1.22 & 0.00146 & 0.62 & 0.00000132 & 0.51 & 0.0000168 \\
\hline K7TTX0 & $\begin{array}{l}\text { Plant UBX domain-containing } \\
\text { protein } 4 \text { OS = Zea mays GN = } \\
\text { ZEAMMB73_Zm00001d025628 }\end{array}$ & 31 & 11.8 & 3 & 4 & 1 & 1.11 & 0.666 & 0.63 & 0.00688 & 0.57 & 0.0355 \\
\hline K7U5A5 & $\begin{array}{l}\text { 14-3-3-like protein OS = Zea } \\
\text { mays GN = ZEAMMB73_ } \\
\text { Zm00001d053090 }\end{array}$ & 193 & 59.7 & 16 & 22 & 11 & 1.42 & 0.00012 & 0.87 & 0.0101 & 0.61 & 0.0000389 \\
\hline K7VEB9 & $\begin{array}{l}\text { Importin subunit alpha OS = Zea } \\
\text { mays GN = ZEAMMB73_- } \\
\text { Zm00001d009850 }\end{array}$ & 29 & 37.8 & 14 & 22 & 2 & 1.41 & 0.0209 & 0.93 & 0.452 & 0.66 & 0.0037 \\
\hline 024415 & $\begin{array}{l}60 S \text { acidic ribosomal protein P2B } \\
\text { OS }=\text { Zea mays } G N=R P P 2 B\end{array}$ & 7 & 10.6 & 1 & 2 & 1 & 1.37 & 0.00026 & 0.90 & 0.00862 & 0.65 & 0.0000431 \\
\hline O64960 & $\begin{array}{l}23.6 \mathrm{kDa} \text { heat shock protein } \\
\text { mitochondrial OS = Zea mays } \\
\mathrm{GN}=\mathrm{hsp} 22\end{array}$ & 18 & 9.2 & 2 & 2 & 2 & 0.65 & 0.00094 & 0.63 & 0.00222 & 0.98 & 0.719 \\
\hline P24631 & $\begin{array}{l}17.5 \mathrm{kDa} \text { class II heat shock } \\
\text { protein OS = Zea mays }\end{array}$ & 43 & 30.4 & 3 & 4 & 3 & 0.52 & $4 \mathrm{E}-05$ & 0.58 & 0.0000602 & 1.11 & 0.00508 \\
\hline
\end{tabular}

Turning to our proteomic data, the proteins involved in alpha-Linolenic fatty acids were up-regulated in V3-6 and V3-6-B treatments. And, the magnitude of the upregulated level was higher in V3-6-B than in V3-6. However, the lipoxygenase (A0A1D6JQF2) and other proteins (acyl carrier protein (B4FDG2 and B6SJF5)) involved in fatty acid biosynthesis pathway were upregulated in V3-6, and down-regulated in V3-6-B. The up-regulation of proteins involved in alpha-Linolenic fatty acids might maintain the plant membrane structure to some extent. However, the increased expression of lipoxygenase (A0A1D6JQF2) might lead to damaged membrane structures and functions. In order to validate the above-mentioned results of protein abundance, the LOX activity and its byproduct $\left(\mathrm{H}_{2} \mathrm{O}_{2}\right.$ and MDA) content were analyzed. The results indicated that LOX activity was significantly increased after waterlogging and the content of its byproducts (MDA and $\mathrm{H}_{2} \mathrm{O}_{2}$ ) were increased correspondingly which hinted damages on membrane structure incurred by waterlogging. However, the activity of LOX and the content of $\mathrm{H}_{2} \mathrm{O}_{2}$ and MDA were effectively reduced after application of 6-BA which supported the results attained by labeling liquid chromatography-based quantitative proteomic analysis and LC-MS/MS. Overall, the present study proved that the waterlogged summer maize plant stimulated some positive response strategies to address waterlogging stress. However, the waterlogging damages were also unequivocally confirmed. Application of 6-BA was conducive to upregulate the proteins (alpha-dioxygenase 1 (A0A1D6P493 and H9BG22) and cytochrome P450 CYP74A19 (A0A096PQR7)) involved in positive response mechanisms of waterlogged plant and downregulate the proteins (LOXs) which do damages on plants. The physiology results (decreased activity of LOX and content of $\mathrm{H}_{2} \mathrm{O}_{2}$ and MDA in V3-6-B) further proved that 6-BA played a pivotal role in reducing the production of ROS by regulating the proteins expression involved in fatty acid metabolism. Spraying 6-BA was conducive to protect the plant membrane from peroxidation damage to some extent.

\section{Proteins involved in protein metabolism}

Protein metabolism processes are closely associated with plant growth and development by regulating a series of biological activities [57]. Zinc finger protein is a transcriptional regulatory factor that regulates gene expression by binding zinc and iron centers to DNA [58, 59], which modulates plant growth and development by regulating gene expression $[60,61]$. It also plays a very important role in plant response to biotic and abiotic stresses [59, 62-64]. In rice, up-regulation of two zinc finger proteins (Q67TK9, Q10N88) and down-regulation of another zinc finger protein (Q5YLY5) contributes to increases of heat tolerance duration at night during grain filling. Our results showed that the RING/FYVE/PHD 


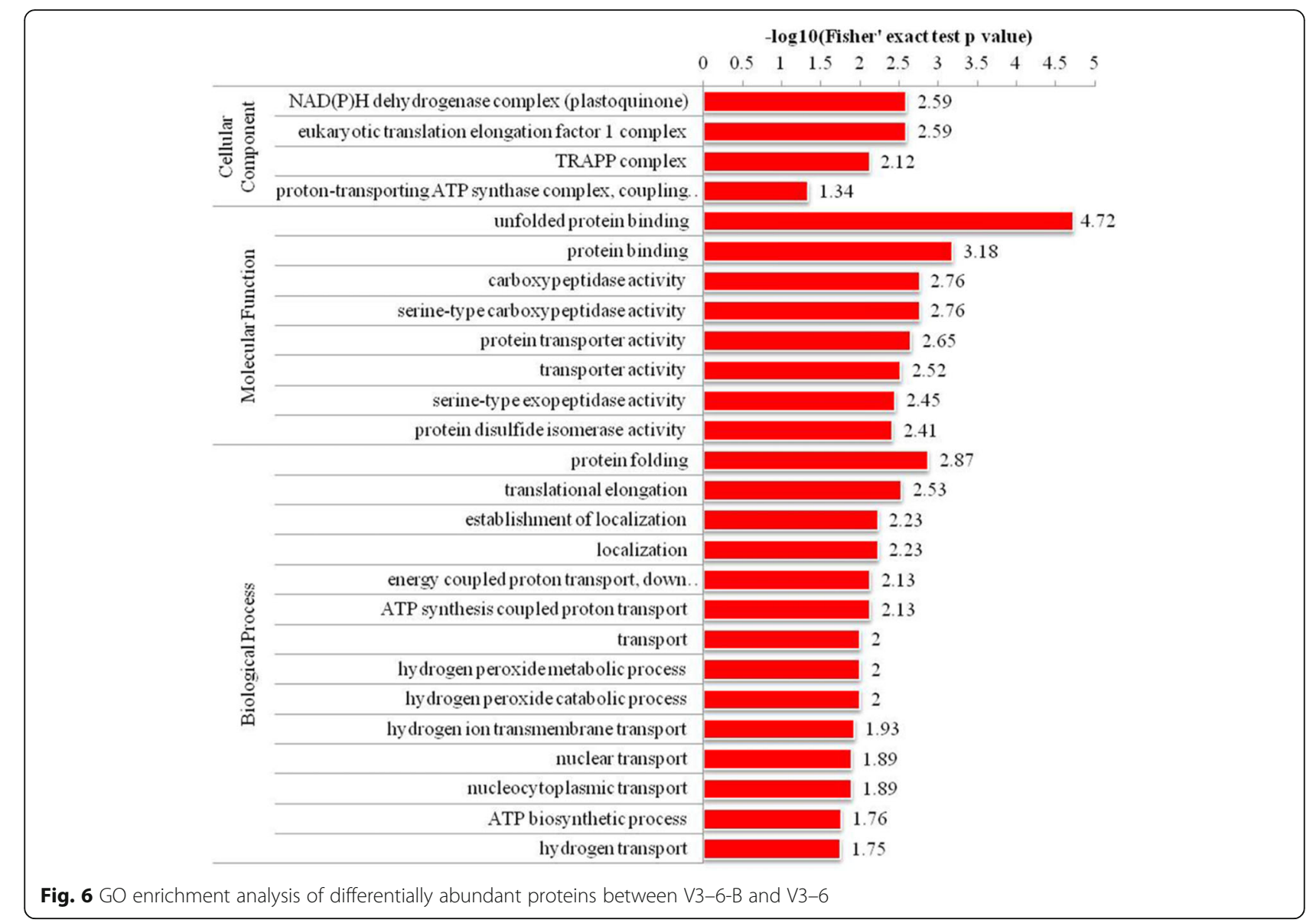

zinc finger superfamily protein (A0A1D6GZM9) was increased in V3-6 treatment and decreased in V3-6-B treatment which might relate with waterlogging tolerance of summer maize. In addition, MBF1 transcription factor (C0P5I3) and NmrA-like negative transcriptional regulator family protein (A0A1D6L6U7) were decreased in both V3-6 and V3-6-B. However, the magnitude of decrease was higher in V3-6 than in V3-6-B. MBF1 transcription factor was a transcriptional Co-activator MBF1c that has a function in thermo-tolerance of Arabidopsis thaliana [65].

NmrA, a negative transcriptional regulator, has function in controlling nitrogen metabolite repression in various fungi by modulating AreA, a kind of GATA-type transcription factor. And, Reiner et al. (2016) proved that a new protein with an NmrA-like domain is involved in cell differentiation and development of Dictyostelium discoideum $[66,67]$. These results suggested that gene involved in stress response or cell development processes were significantly down regulated in V3-6 which might be responsible for the intolerance to waterlogging of summer maize. However, Compared with $\mathrm{CK}$, proteins involved in translation process, such as nucleosome assembly protein 1 (COHF19 and
A0A1D6MBB0), elongation factor 1-beta (B6SLK4 and B4FNT1), 30S ribosomal protein S1 (B4FUZ5), 60S ribosomal protein L22-2 (B4FCK9), and nucleic acidbinding OB-fold-like proteins (A0A1D6H4K9 and B4FT80) were significantly increased in V3-6, while spraying 6-BA was effectively conducive to mitigate these increases.

Most proteins are required to fold into fine threedimensional structures to perform functional activity. However, many proteins fold aberrantly and even aggregated under stress conditions [68, 69]. Protein aberrant folding and aggregation occurred with an increasing frequency under environmental stresses such as high temperature and drought [70]. Therefore, plant needs to evolve numerous mechanisms to address aberrant folding and aggregation. Hsp90 and small Hsps have been demonstrated to have functions in preventing aggregation and promoting efficient folding under adverse conditions [71]. Recent research also demonstrated that drought aroused a remarkable increase of some sHSPs in maize leaves [72]. In our study, Hsps (O64960, B6TDB5, and P24631) were significantly down-regulated in waterlogged plant leaves, while 6-BA mitigated these decreases. However, the key constituents of the 


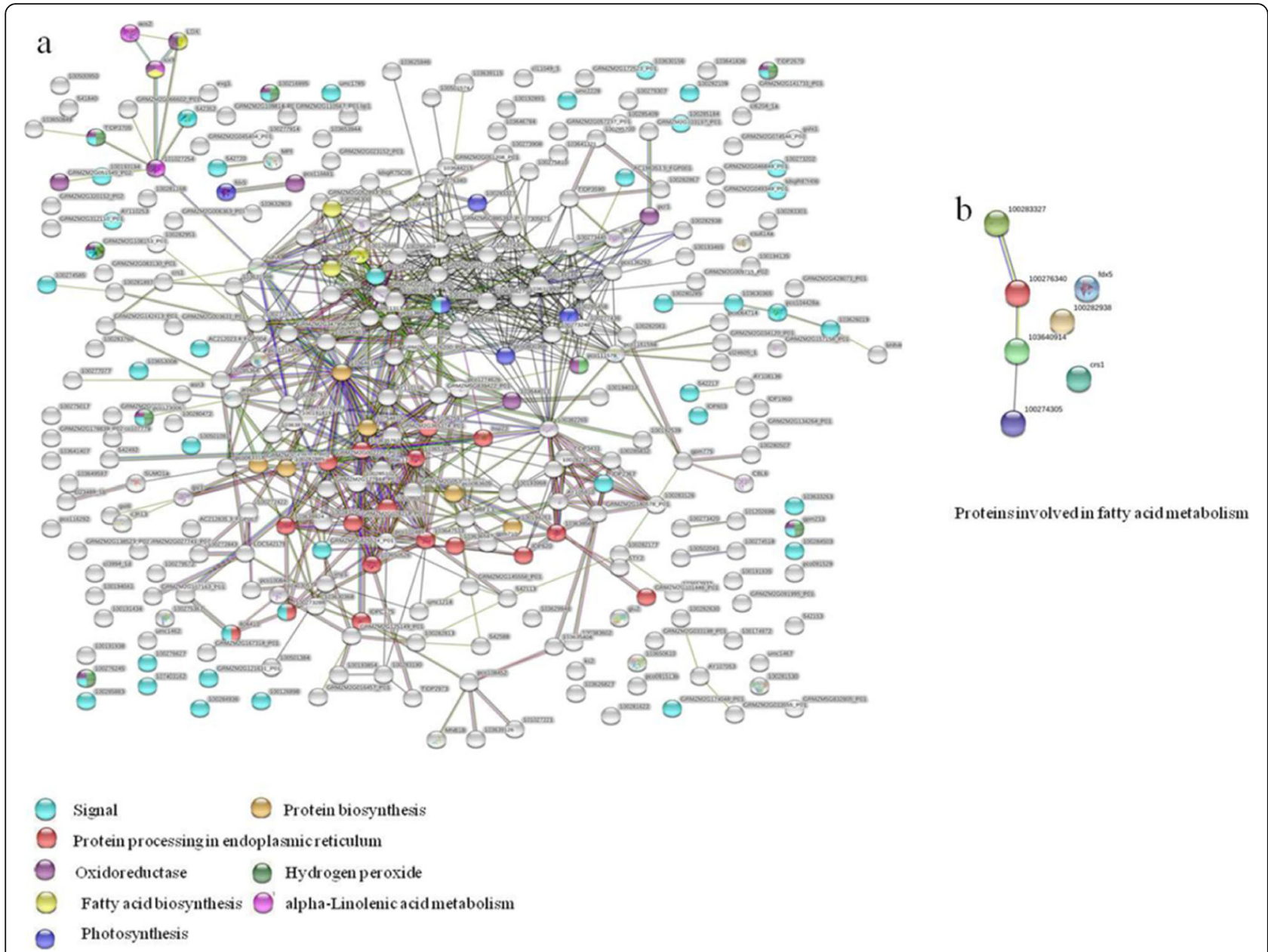

Fig. 7 The protein-protein interaction network of the differentially abundant proteins, a The protein-protein interaction network of the overall differentially abundant proteins, b Proteins involved in fatty acid metabolism

degradation system, 26S proteasome subunits (A0A1D6HCE3, B6T9G1, A0A1D6KA58, and A0A1D6GTD7), were up-regulated in waterlogged plants whereas no significant changes were observed in the V3-6-B treatment. In addition, proteins associated with unfolded protein binding, phosphatase, proteolysis, and ubiquitination (A0A1D6E942 and B4FUZ5; B6UG10; A0A1D6H5B6, B4FKB3) were decreased greatly in V3-6. These results suggested waterlogging affected the protein folding and other processing processes which impeded the proteins to perform functions. However, application of 6-BA was conducive to mitigate waterlogging impacts on these proteins.

These results indicated that waterlogging triggered notable changes in protein metabolism process including protein synthesis, protein processing, protein homeostasis and degradation processes. The extensive changes in protein metabolism process might result in disorders of cell metabolism activities such as substance synthesis, transport, and secondary metabolism processes. In our previous study, carbon and nitrogen metabolism processes were significantly affected by waterlogging, which disturbed the balance of cell activities, affecting substance synthesis and transport, and thus limited plant growth. Impediment of plant growth induced by waterlogging was also observed in this study, while spraying 6-BA mitigated this impediment $[26,73]$.

Overall, the present study proved that 6-BA increased the defense system activity by modulating the expression of proteins related with ROS and fatty acid metabolism which worked together to maintain the integrity of cell membranes. Conversely, the increased integrality of cell membranes improved the protein metabolism processes which might be responsible for the improvement of plant growth rate and yield of waterlogged summer maize.

\section{Conclusions}

Based on our study, we demonstrated that 6-BA had contrastive effects on waterlogged summer maize (Fig. 8). 


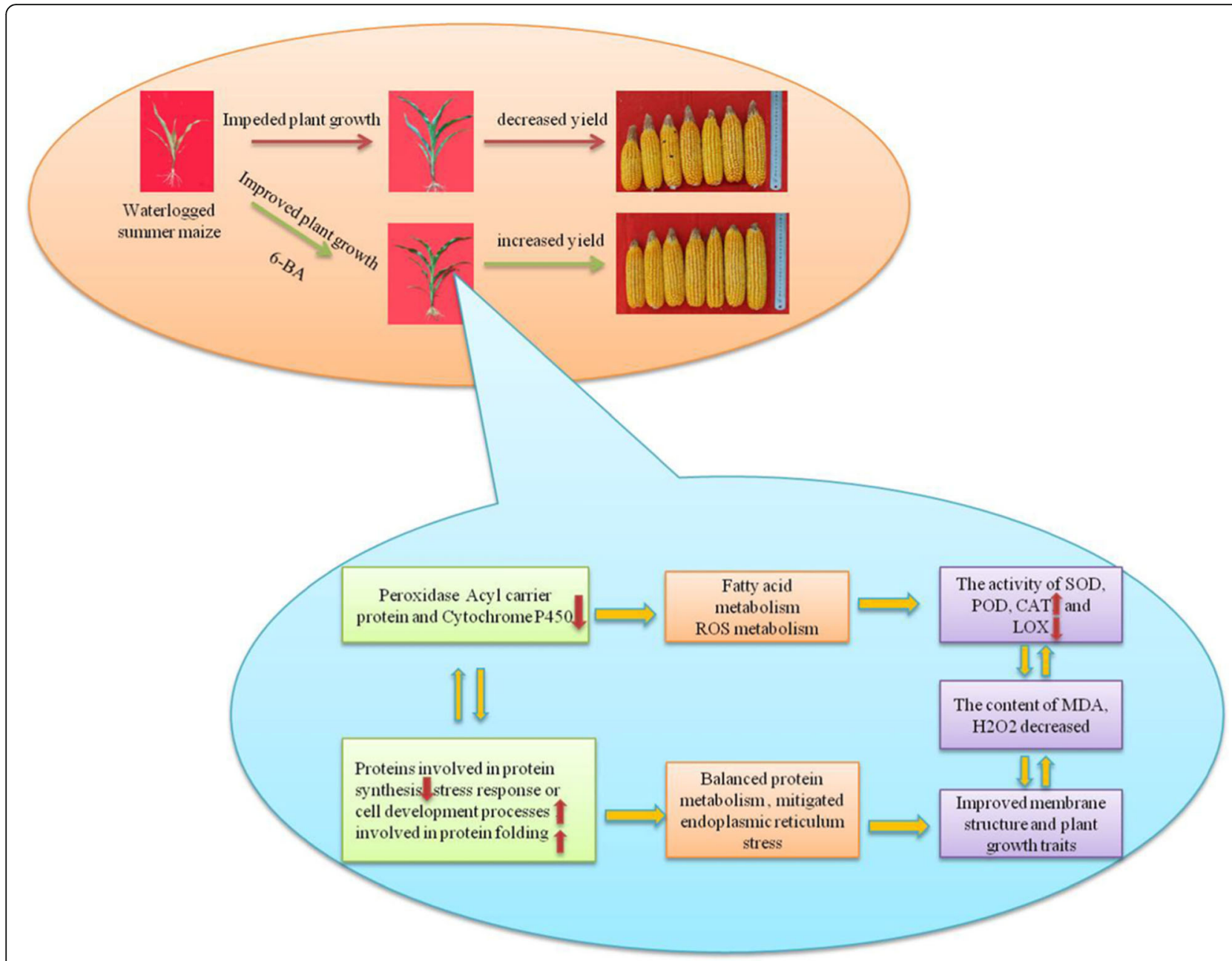

Fig. 8 Exogenous 6-benzyladenine (6-BA) improve the defense system activity of waterlogged summer maize

Waterlogging caused significant impediment of plant growth and decreased the activities of SOD, POD and CAT of summer maize. In addition, the activity of LOX was significantly increased. As a result, the content of MDA and $\mathrm{H}_{2} \mathrm{O}_{2}$ was significantly increased which incurred serious damages on cell membrane and cellular metabolism. In addition, the plant growth rate and grain yield were significantly decreased by waterlogging. However, application of 6-BA effectively mitigated these adverse effects induced by waterlogging. Based on proteomics profiling, the proteins involved in protein metabolism, ROS scavenging and fatty acid metabolism were significantly regulated by 6 -BA. The results suggested that application of 6-BA exaggerated the defensive response of summer maize at proteomic level leading to improved plant growth traits and a higher grain yield. Although further studies are required to investigate the regulatory mechanism of 6-BA on waterlogged summer maize, the results in our study provide a foundation for further researches.

\section{Methods}

\section{Experimental design}

The field experiment was performed at the experimental farm of Shandong Agricultural University, China $\left(36^{\circ}\right.$ $\left.10^{\prime} \mathrm{N}, 117^{\circ} 09^{\prime} \mathrm{E}\right)$. This study was conducted in accordance with local legislations and obtained the permission from Shandong Agricultural University complying with the Convention on the Trade in Endangered Species of Wild Fauna and Flora. The summer maize hybrid DengHai605 (DH605) was used in this study. DH605 is the hybrid of DH351 (selected from a self-breeding multiple generation of DH158/107) and DH382 (selected from a self-breeding multiple generation of American germplasm X1132) bred by Shandong Denghai Seeds Co., Ltd. It was approved by the National crop variety examination and approval committee of china on $09^{\text {th }}$ 2010 which suitable for planting in Shandong province and most part of china. Maize was sown on June with a plant density of 67,500 plants ha ${ }^{-1}$ and well managed. Three experimental treatments were set: control (CK), 
waterlogging at the third leaf stage for 6 days (V3-6) and $100 \mathrm{mg} \mathrm{dm}^{-3}$ 6-BA was applied after waterlogging (V3-6-B). Three replicated fresh ear leaf tissue were sampled at tasseling stage from each treatment and frozen in liquid nitrogen and kept at $-80^{\circ} \mathrm{C}$ until further use. No permissions or licenses were needed to obtain our plant sample.

\section{Physiological traits determination \\ Antioxidant enzyme activity and MDA content}

The middle of functional leaf from three plant samples was obtained to determine the protective enzymes activity and MDA content. The activity of SOD, POD, and CAT was assayed according to the method described by Giannopolitis and Ries (1977), Hammerschmidt et al. (1982) and Durner and Klessing (1996), respectively [74-76]. In addition, the MDA content was determined according to Du et al. (1992) [77]. Three biological replicates of the individual samples were used for analysis. Oneway ANOVA and comparisons between means were made following the LSD test at $P<0.05$ and performed with SPSS V.21.0.

\section{Estimation of LOX activity and $\mathrm{H}_{2} \mathrm{O}_{2}$ content}

The LOX activity and $\mathrm{H}_{2} \mathrm{O}_{2}$ content were determined according to the method described by $\mathrm{Fu}$ et al. (1996) [78] and Bizzi et al. [79], respectively. One-way ANOVA and comparisons between means were made following the LSD test at $P<0.05$ and performed with SPSS V. 21.0.

\section{Plant growth rate and yield}

The number of the fully extended leaves was observed at V6, V9, V12 growth stages (based on 50\% of all plants in the plot attaining the growth stage) of CK. Fifteen representative plant height was measured at VT. Thirty ears from each plot were harvested to calculate yield at R6. One-way ANOVA and comparisons between means were made following the LSD test at $P<0.05$ and performed with SPSS V. 21.0.

\section{Proteomic analysis}

The leaf samples of three plants from each treatment were mixed and exposed to liquid nitrogen, ground into cell powder and then four volumes of lysis buffer $(8 \mathrm{M}$ urea, $1 \%$ Protease Inhibitor Cocktail) were added to the cell powder for extracting proteins. Then, the extracted protein solutions were digested by trypsin. After digestion, the peptides were labeled by TMT then for HPLC fractionation using Thermo Betasil C18 column $(5 \mu \mathrm{m}$ particles, $10 \mathrm{~mm}$ ID, $250 \mathrm{~mm}$ length; Bellefonte, PA, USA) and LC-MS/MS analysis using Orbitrap FusionTM (ThermoFisher Scientific, Waltham, USA). The MS/MS data were searched against UniProt Zea mays L database
(99,450 sequences, 201,706) concatenated with reverse decoy database using the Maxquant search engine (v.1.5.2.8). Trypsin/P was specified as a cleavage enzyme allowing up to 2 missing cleavages. The mass tolerances for precursor ions in First search and Main search were set as $20 \mathrm{ppm}$ and $5 \mathrm{ppm}$, respectively. $0.02 \mathrm{Da}$ was set as the mass tolerance for fragments. Carbamidomethyl on Cys and oxidation on Met (or Acetylation on the Nterminal) were specified as fixed modification and variable modifications, respectively. False discovery rate (FDR) was set $<1 \%$ and minimum score for peptides was set $>40$ to assess the confidence of the peptides [80]. TMT 10-plex was used in Mascot to quantify identified proteins. And, proteins with $P$-value $<0.05$ and fold changes $>1.5$ or $<0.667$ were considered as significant differentially abundant. After screening for differentially abundant multiples, bioinformatics analysis including protein annotation, functional classification, functional enrichment, and cluster analysis of differentially abundant proteins were performed. The Gene Ontology functional annotations (GO) and Kyoto Encyclopedia of Genes and Genomes analysis (KEGG) of identified proteins were searched against GO database (www.http://www.ebi.ac.uk/GOA/) and KEGG database (http://www.genome.jp/kegg/pathway.html) using Blast2GO program (https://www.blast2go.com/) and Blastx/ Blastp 2.2.24 software, respectively. Wolfpsort (an updated version of PSORT/PSORT II) was used to predict subcellular localization. Then, GO and KEGG pathway enrichment analysis of the DAPs were implemented with a $P$-value $<0.05$ [81]. Three technical replicates of each sample were performed. This proteomic analyses was provided by Jingjie PTM BioLabs, Inc. The more detailed method was described in Additional file 4.

\section{Supplementary information}

Supplementary information accompanies this paper at https://doi.org/10. 1186/s12870-020-2261-5.

Additional file 1: Quality detection of mass spectrometry and part of results, Figure S1. The distribution of peptide mass error and peptide length. Figure S2. The Pearson's correlation of quantitation among treatments. Figure S3. The differentially abundant proteins among treatments.

Additional file 2. GO term level2 classification of differentially abundant proteins.

Additional file 3. Details of differentially abundant proteins.

Additional file 4. Details of MS Methods.

\section{Abbreviations}

6-BA: 6-benzyladenine; ANOVA: Analysis of variance; CAT: Catalase; CK: Control; Cys: Cysteine; DAPs: Differentially abundant proteins; DH605: Summer maize hybrid DengHai 605; FDR: False discovery rate; GO: Gene Ontology; $\mathrm{H}_{2} \mathrm{O}_{2}$ : Hydrogen peroxide; HPLC: High-pressure liquid chromatography; KEGG: Kyoto Encyclopedia of Genes and Genomes; LC: Liquid chromatography; LOX: Lipoxygenase; LSD: Least significant difference; MDA: Malondialdehyde; MS: Mass spectrometry; POD: Peroxidase; 
ROS: Reactive oxygen species; SOD: Superoxide dismutase; TEAB: Tetraethylammonium bromide; TMT: Tandem Mass Tags; V12: Twelfth leaf stage; V3-6: Waterlogging at the third leaf stage for 6 days; V3-6B: Application of $100 \mathrm{mg} \mathrm{dm}^{-3}$ 6-BA after waterlogging for 6 days; V6: Sixth leaf stage; V9: Ninth leaf stage; VT: Tasseling stage

\section{Acknowledgments}

We thank PTM Biolabs, Inc. for his advice on bioinformatics.

\section{Authors' contributions}

$\mathrm{JZ}$ conceived and designed the experiments; JH performed the experiments and drafted the manuscript; BR and BZ made substantial contributions to conception and experiment design, BR, SD and PL helped with discussion and revised the manuscript. All authors have read and approved the manuscript.

\section{Funding}

This study was funded by National Key Research and Development Program of China (2017YFD0300304, 2018YFD0300603), National Nature Science Funds (31671629, 31801296), National Modern Agricultural Technology \& Industry System (CARS-02-18), Shandong "Double Tops" Program Funds (SYL2017YSTD02). The funding bodies did not play a role in the design research, measurements, data analysis, and drafted the manuscript, but just provide the financial support.

\section{Availability of data and materials}

The data supporting the conclusions of this article are included within the article and its additional files. And also, the mass spectrometry protemics datasets have been deposited to the ProteomeXchange Consortium via the PRIDE [82] partner repository with the dataset identifier PXD016743", (https:// www.ebi.ac.uk/pride/profile/reviewer85471).

\section{Ethics approval and consent to participate}

$$
\text { Not applicable. }
$$

\section{Consent for publication}

Not applicable.

\section{Competing interests}

The authors declare that they have no competing interests.

\section{Received: 20 November 2019 Accepted: 20 January 2020}

\section{Published online: 29 January 2020}

\section{References}

1. Solomon S, Qin DH, Manning M, Chen Z, Marquis M, Tignor M, Averyt KB. Climate change 2007: the physical science basis. Contribution of working group I to the fourth assessment report of the intergovernmental panel on climate change. Summary for policymakers. Intergov Panel Climate Change Climate Change. 2007:18:95-123.

2. Liu JF, Wang JH, Jiao MH, Zhang RG. Response of water resources in the yellow river basin to global climate change. Arid Zone Res. 2011;28:860-5.

3. Edenhofer O, Seyboth K. Intergovernmental panel on climate change (IPCC). Encyclopedia Energy Nat Res Environ Econ. 2013;26:48-56.

4. Lal R. Soil carbon sequestration to mitigate climate change. Geoderma. 2004;123:0-22.

5. Qin XS, Lu Y. Study of climate change impact on flood frequencies: a combined weather generator and hydrological modeling approach. J Hydrometeorol. 2014;15:1205-19.

6. Li X, Zhang JW, Ren BZ, Fan X, Zhao B. Effects of tillage at pre-planting of winter wheat and summer maize on leaf senescence of summer maize. J Appl Ecol. 2015;26:1397-403

7. Warren RF, Wilby RL, Brown K, Watkiss P, Betts RA, Murphy JM. Advancing national climate change risk assessment to deliver national adaptation plans. Philos Transact Royal Soc Math Phys Eng Sci. 2018;376:1-28.

8. Bruckner T, Petschel-Held G, Tóth FL, Füssel HM, Helm C, Leimbach M. Climate change decision-support and the tolerable windows approach. Environ Modeling Assess. 1999:4:217-34.

9. Yu WD, Liu J, Chang J, Wang J. Changes in extreme temperature and precipitation in Henan province during 1957-2005. Adv Clim Chang Res. 2008;2:1673-719.
10. Islam MM, Jannat A, Dhar AR, Ahamed T. Factors determining conversion of agricultural land use in Bangladesh: farmers' perceptions and perspectives of climate change. Geo J. 2019;3:1-20.

11. Ren BZ, Zhang JW, Li X, Fan X, Dong ST, Liu P. Effects of waterlogging on the yield and growth of summer maize under field conditions. Revue Canadienne De Phytotechnie. 2014;94:23-31.

12. Zaidi PH, Rafique S, Rai PK, Singh NN, Srinivasan G. Tolerance to excess moisture in maize (Zea mays L.): susceptible crop stages and identification of tolerant genotypes. Field Crop Res. 2004;90:0-202.

13. Duan B, Yang $Y$, Lu Y, Korpelainen H, Berninger F, Li C. Interactions between drought stress, $A B A$ and genotypes in Picea asperata. J Exp Bot. 2007:58:3025-36.

14. Yan B, Dai Q, Liu X, Huang S, Wang Z. Flooding-induced membrane damage, lipid oxidation and activated oxygen generation in corn leaves. Plant and Soil. 1996:179:261-8.

15. Anjum S, Xie X, Wang L, Saleem M, Man C, Lei W. Morphological, physiological and biochemical responses of plants to drought stress. Afr J Agric Res. 2011;6:2026-32.

16. Bowler C, Montagu MV, Inze D. Superoxide dismutase and stress tolerance. Annu Rev Plant Physiol Plant Mol Bol. 1992;43:83-116.

17. Hodgson RAJ, Raison JK. Superoxide production by thylakoids during chilling and its implication in the susceptibility of plants to chilling-induced photoinhibition. Planta. 1991;183:222-8.

18. Hurng WP, Kao CH. Lipid peroxidation and antioxidative enzymes in senescing tobacco leaves during post-flooding. Plant Sci. 1994;96:41-4.

19. Price AH, Atherton NM, Hendry GAF. Plants under drought-stress generated activated oxygen. Free Radic Res Commun. 1989;8:61-6.

20. Zhang JX, Kirkham MB. Drought-stress-induced changes in activities of superoxide dismutase, catalase, and peroxidase in wheat species. Plant Cell Physiol. 1994;35:785-91.

21. Ádám A, Farkas T, Somlyai G, Hevesi M, Király Z. Consequence of $\mathrm{O}^{2-}$ generation during a bacterially induced hypersensitive reaction in tobacco: deterioration of membrane lipids. Physiol Mol Plant Pathol. 1989;34:13-26.

22. Ding $X T$, Jiang $Y P$, Wang $H$, Chen $\mathrm{CH}$. Effects of cytokinin on photosynthetic gas exchange, chlorophyll fluorescence parameters, antioxidative system and carbohydrate accumulation in cucumber (Cucumis sativus L.) under low light. Acta Physiol Plant. 2003;35:1427-38.

23. Tang B, Xu SZ, Zou XL, Zheng YL, Qiu FZ. Changes of antioxidative enzymes and lipid peroxidation in leaves and roots of waterloggingtolerant and waterlogging-sensitive maize genotypes at seedling stage. J Integr. 2010;9:0-661.

24. Majid GJ, Ali S, Seyed AM, Iraj A, Foad M. Effects of the exogenous application of auxin and cytokinin on carbohydrate accumulation in grains of rice under salt stress. Plant Growth Regul. 2011;65:305-13.

25. Nurunnaher A, Rafiqul IM, Abdul KM, Tofazzal H. Alleviation of drought stress in maize by exogenous application of gibberellic acid and cytokinin. J Crop Sci Biotechnol. 2014;17:41-8.

26. Ren BZ, Zhang JW, Dong ST, Liu P, Zhao B. Regulations of 6-benzyladenine (6-BA) on leaf ultrastructure and photosynthetic characteristics of waterlogged summer maize. J Plant Growth Regul. 2017;36:743-54.

27. Wijk KJV. Challenges and prospects of plant proteomics. Plant Physiol. 2001; 126:501-8.

28. Morel J. Proteomics of plant detergent-resistant membranes. Mol Cell Proteomics. 2006;5:1396-411.

29. Reddy VS, Reddy ASN. Review proteomics of calcium-signaling components in plants. Phytochemistry. 2004;65:1745-76.

30. Zhang T, Zhao X, Wang W, Pan Y, Huang L, Liu X. Comparative transcriptome profiling of chilling stress responsiveness in two contrasting rice genotypes. PLoS One. 2012;7:153-4.

31. Zhang Z, Zhang Y, Liu X, Li Z, Lin W. The use of comparative quantitative proteomics analysis in rice grain-filling in determining response to moderate soil drying stress. Plant Growth Regul. 2017;82:1-14.

32. Obata T, Fernie AR. The use of metabolomics to dissect plant responses to abiotic stresses. Cell Mol Life Sci. 2012;69:3225-43.

33. Maffei ME, Mithofer A, Boland W. Insects feeding on plants: rapid signals and responses preceding the induction of phytochemical release Phytochemistry. 2007;68:2946-59.

34. War AR, Paulraj MG, War MY, Ignacimuthu S. Jasmonic acid-mediated induced resistance in groundnut (Arachis hypogaea L.) against Helicoverpa armigera (Hubner) (Lepidoptera: Noctuidae). J Plant Growth Regul. 2011;30:512-23. 
35. War AR, Paulraj MG, War MY, Ignacimuthu S. Herbivore- and Elicitor-induced Resistance in Groundnut to Asian armyworm, Spodoptera litura (Fab.) (Lepidoptera: Noctuidae). Plant Signal Behav. 2011;6:1769-77.

36. Kawano T. Roles of reactive oxygen species - generating peroxidase reactions in plant defense and growth induction. Plant Cell Rep. 2003;21:829-37.

37. War AR, Paulraj MG, War MY, Ignacimuthu S. Differential defensive response of groundnut to Helicoverpa armigera (Hubner) (Lepidoptera: Noctuidae). J Plant Interact. 2011;6:1-11.

38. Chen Y, Ni X, Buntin GD. Physiological, nutritional and biochemical bases of corn resistance to foliage-feeding fall armyworm. J Chem Ecol. 2009;35:297-306.

39. Asada K. Production and scavenging of reactive oxygen species in chloroplasts and their functions. Plant Physiol. 2006;141:391-6.

40. Dean RT, Fu S, Stocker R, Davies MJ. Biochemistry and pathology of radicalmediated protein oxidation. Biochem J. 1997;324:1-18.

41. Berlett BS. Protein oxidation in aging, disease, and oxidative stress. J Biol Chem. 1997;272:20313-6.

42. Xu QT, Yang L, Zhou ZQ, Mei FZ, Qu LH, Zhou GS. Process of aerenchyma formation and reactive oxygen species induced by waterlogging in wheat seminal roots. Planta. 2013;238:969-82.

43. Agarwal S, Sairam RK, Srivastava GC, Meena RC. Changes in antioxidant enzymes activity and oxidative stress by abscisic acid and salicylic acid in wheat genotypes. Biol Plant. 2005;49:541-50.

44. Xu X, Ji J, Xu Q, Qi X, Chen X. The major-effect QTL csarn6.1 encodes AAAATPase domain-containing protein that is associated with waterlogging stress tolerance through promoting adventitious root formation. Plant J. 2018;93:917-30.

45. Bóka K, Orbán N, Kristóf Z. Dynamics and localization of h2o2production in elicited plant cells. Protoplasma. 2007;230:89-97.

46. Apel K, Hirt H. Reactive oxygen species: metabolism, oxidative stress, and signal transduction. Annu Rev Plant Biol. 2004;55:373-99.

47. Gopala Krishna J, Gandhi KRR. General, one \& several variable extensions of chinese remainder theorem (additional features with co-primality conditions). Int.j.math.sci.appl. 2011;1:1215-23.

48. Liu W, Wang LH, Fabian P, Hayashi Y, Mcginley CM, Donk WAVD. Arabidopsis thaliana fatty acid alpha-dioxygenase-1: evaluation of substrates, inhibitors and amino-terminal function. Plant Physiol Biochem (Paris). 2006:44:284-93.

49. Li L, Chang Z, Pan Z, Fu ZQ, Wang X. Modes of heme binding and substrate access for cytochrome p450 cyp74a revealed by crystal structures of allene oxide synthase. Proc Natl Acad Sci U S A. 2008;105:13883-8.

50. Somerville C, Browse J. Plant lipids: metabolism, mutants, and membranes Science. 1991;252:80-7

51. Kirsch C. Rapid and transient induction of a parsley microsomal delta 12 fatty acid desaturase mRNA by fungal elicitor. Plant Physiol. 1997;115:283-9.

52. Browse J, Mcconn M, James D, Miquel M. Mutants of arabidopsis deficient in the synthesis of alpha-linolenate biochemical and genetic characterization of the endoplasmic reticulum linoleoyl desaturase. J Bio Chem. 1993;268:16345-51.

53. Miquel M, Browse J. Arabidopsis mutants deficient in polyunsaturated fatty acid synthesis: biochemical and genetic characterization of a plantoleoylphosphatidylcholine desaturase. J Biol Chem. 1992;267:1502-9.

54. Maalekuu K, Elkind Y, Leikin-Frenkel A, Lurie S, Fallik E. The relationship between water loss, lipid content, membrane integrity and lox activity in ripe pepper fruit after storage. Postharvest Biol Technol. 2006;42:248-55.

55. Siedow JN. Plant lipoxygenase: structure and function. Annu Rev Plant Physiol Plant Mol Biol. 1991;42:145-88.

56. Glickman MH, Klinman JP. Lipoxygenase reaction mechanism: demonstration that hydrogen abstraction from substrate precedes dioxygen binding during catalytic turnover. Biochemistry. 1996;35:12882-92.

57. Swidzinski JA, Leaver CJ, Sweetlove LJ. A proteomic analysis of plant programmed cell death. Phytochemistry. 2004;65:1829-38.

58. Klug A. The discovery of zinc fingers and their development for practical applications in gene regulation and genome manipulation. Annu Rev Biochem. 2010;43:1-21.

59. Zhang $Y$, Lan $H$, Shao Q, Wang $R$, Chen $H$, Tang $H$. An a20/an1-type zinc finger protein modulates gibberellins and abscisic acid contents and increases sensitivity to abiotic stress in rice (Oryza sativa). J Exp Bot. 2016;67:315-26.

60. Chen Y, Sun A, Wang M, Zhu Z, Ouwerkerk PBF. Functions of the CCCH type zinc finger protein OsGZF1 in regulation of the seed storage protein Glub-1 from rice. Plant Mol Biol. 2013;84:621-34.
61. Laity $\mathrm{JH}$, Lee $B M$, Wright PE. Zinc finger proteins: new insights into structural and functional diversity. Curr Opin Struct Biol. 2001;11:39-46.

62. Wang F, Tong W, Zhu H, Kong W, Peng R, Liu Q. A novel Cys2/His2zinc finger protein gene from sweet potato, IbZFP1, is involved in salt and drought tolerance in transgenic Arabidopsis. Planta. 2016;243:783-97.

63. Huang XY, Chao DY, Gao JP, Zhu MZ, Shi M, Lin HX. A previously unknown zinc finger protein, DST, regulates drought and salt tolerance in rice via stomatal aperture control. Genes Dev. 2009;23:1805-17.

64. Yue XF, Que YW, Xu ML, Deng SZ, Peng MY, Talbot N. ZNF1 encodes a putative $\mathrm{C} 2 \mathrm{H} 2$ zinc finger protein essential for appressorium differentiation by the rice blast fungus magnaporthe oryzae. Mol Plant-Microbe Interact. 2015;29:22-35

65. Suzuki N, Bajad S, Shuman J, Shulaev V, Mittler R. The transcriptional coactivator $\mathrm{mbf1c}$ is a key regulator of thermotolerance in arabidopsis thaliana. J Biol Chem. 2008;283:9269-75.

66. Beatriz N-C, Serafimidis I, Arias-Palomo E, Rivera-Calzada A, Suarez T. A new protein carrying an nmra-like domain is required for cell differentiation and development in dictyostelium discoideum. Dev Biol. 2008;321:0-342.

67. Reiner T, Hoefle C. Ralph Hückelhoven. A barley skp1-like protein controls abundance of the susceptibility factor racb and influences the interaction of barley with the barley powdery mildew fungus. Mol Plant Pathol. 2016;17:184-95.

68. Hartl FU, Bracher A, Hayer-Hartl M. Molecular chaperones in protein folding and proteostasis. Nature. 2011;475:324-32.

69. Kim YE, Hipp MS, Bracher A, Hayer-Hartl M, Hartl FU. Molecular chaperone functions in protein folding and proteostasis. Annu Rev Biochem. 2013;82:323-55.

70. Dobson CM. Protein folding and misfolding. Am Sci. 2003;426:884-90.

71. Jakob U, Buchner J. Assisting spontaneity: the role of hsp90 and small hsps as molecular chaperones. Trends Biochem Sci. 1994;19:205-11.

72. Yang L, Jiang T, Fountain JC, Scully BT, Lee RD, Kemerait RC. Protein profiles reveal diverse responsive signaling pathways in kernels of two maize inbred lines with contrasting drought sensitivity. Int J Mol Sci. 2014;5:18892-918.

73. Ren BZ, Zhu Y, Zhang J, Dong S, Liu P, Zhao B. Effects of spraying exogenous hormone 6-benzyladenine (6-BA) after waterlogging on grain yield and growth of summer maize. Field Crop Res. 2016;188:96-104.

74. Giannopolitis CN, Ries SK. Superoxide Dismutases I. Occurrence in higher plants. Plant Physiol. 1977;59:309-14.

75. Hammerschmidt R. Association of enhanced peroxidase activity with induced systemic resistance of cucumber to colletotrichum lagenarium. Physiol Plant Pathol. 1982;20:77-6.

76. Durner J, Klessig DF. Salicylic acid is a modulator of tobacco and mammalian catalases. J Biol Chem. 1996;271:28492-501.

77. Du ZY, Bramlage WJ. Modified thiobarbituric acid assay for measuring lipid oxidation in sugar-rich plant tissue extracts. J Agric Food Chem. 1992;40:1566-70.

78. Fu C, Xu W, Chang R, Ding A. Identification of the heterogenity and analysis of the activity of chinese soybean lipoxygenase. Acta Agriculturae Boreall-sinica. 1996;11:25-9.

79. Bizzi CA, Flores EL, Nóbrega JA, Oliveira JSS, Schmidt L, Mortari SR. Evaluation of a digestion procedure based on the use of diluted nitric acid solutions and $\mathrm{H}_{2} \mathrm{O} 2$ for the multielement determination of whole milk powder and bovine liver by ICP-based techniques. J Anal At Spectrom. 2014;29:332-8.

80. Ma YZ, Wang KZ, Pan JB, Fan ZH, Tian CH, Deng XB. Induced neural progenitor cells abundantly secrete extracellular vesicles and promote the proliferation of neural progenitors via extracellular signal-regulated kinase pathways. Neurobiol Dis. 2019;124:322-34.

81. Yu RG, Jiang $Q, X v C$, Li L, Bu SJ, Shi GR. Comparative proteomics analysis of peanut roots reveals differential mechanisms of cadmium detoxification and translocation between two cultivars differing in cadmium accumulation. BMC Plant Biol. 2019;19:137.

82. Deutsch EW, Csordas A, Sun Z, Jarnuczak A, Perez-Riverol Y, Ternent T, Campbell DS, Bernal-Llinares M, Okuda S, Kawano S, Moritz RL, Carver Jر, Wang M, Ishihama Y, Bandeira N, Hermjakob H, Vizcaíno JA. The ProteomeXchange consortium in 2017: supporting the cultural change in proteomics public data deposition. Nucleic Acids Res. 2017;54(D1):D1100-6 PubMed PMID: 27924013.

\section{Publisher's Note}

Springer Nature remains neutral with regard to jurisdictional claims in published maps and institutional affiliations. 